Vietnam Academy of Science and Technology
(VAST)

\title{
Petrography and geochemistry of Permian basalts of the Cam Thuy formation and their relation to Song $\mathrm{Da}$ and Emeishan magmatic rocks
}

\author{
Nguyen Hoang ${ }^{1 *}$, Tran Thi Huong', Masatsugu Ogasawara², Le Duc Anh ${ }^{3}$, \\ Nguyen Thi Mai' ${ }^{1}$ Nguyen Thi Thu', Cu Sy Thang', Le Thi Phuong Dung ${ }^{1}$ \\ ${ }^{1}$ Institu te of Geological Sciences, Vietnam Academy of Science and Technology (VAST), Hanoi, Vietnam \\ ${ }^{2}$ Geological Survey of Japan, National Institute of Advanced Industrial Science and Technology (AIST), Tsukuba, Japan \\ ${ }^{3}$ Institute of Marine Geology and Geophysics (VAST), Hanoi, Vietnam
}

Received 20 June 2015. Accepted 14 December 2016

\begin{abstract}
Cam Thuy Permian basalts consisting of thick lava flows and pyroclastic layers appear along both sides of the Song Ma fault zone in Thanh Hoa and in Son La and Ninh Binh provinces, NW Vietnam. The magmatism has been thought to have genetic relationship with Permian volcanism in the Song Da rift zone, which is believed to be part of the Emeishan large igneous province, having been extruded along the Red River shear zone following Paleogene India-Eurasian collision. A set of Cam Thuy volcanic samples including olivine and alkaline basalts was collected in the Lam Son area (Tho Xuan, Thanh Hoa province) to analyze for geochemical major, trace element and Sr- $\mathrm{Nd}-\mathrm{Pb}$ isotopic composition. The Cam Thuy basalts are high- $\mathrm{TiO}_{2}, \mathrm{CaO}, \mathrm{FeO} *$, moderate $\mathrm{MgO}$ and $\mathrm{SiO}_{2}$ that plot between the Song Da and Emeishan high- and low-Ti basalt distribution fields and closely overlap that of Song Da's high-Ti field. The primitive mantle and chondrite normalized trace element patterns of Cam Thuy basalts are essentially enriched oceanic island basalt (OIB)-like; this feature, together with crustal contamination-free, chondritic $\mathrm{Sr}, \mathrm{Nd}$ and $\mathrm{Pb}$ initial (255Ma) isotopic composition are certainly of asthenospheric origin. These geochemical and isotopic characteristics are closely analogous to those features observed for the Song Da high-Ti basalts, suggesting similarity in their source of origin. Nevertheless, while the Song Da (and Emeishan) magmatism is signified by the presence of both high- and low-Ti basalts, with the latter being derived from heterogeneous and partially crustal-material contaminated sources in the lithospheric mantle, this low-Ti volcanic rock type has yet to be discovered in the Cam Thuy formation.
\end{abstract}

Keywords: Northwest Vietnam, Song Ma fault zone, Song Da Rift zone, Cam Thuy Permian basalt, isotopic geochemistry.

(C)2016 Vietnam Academy of Science and Technology

\section{Introduction}

The Song Da structure, viewed as a

*Corresponding author, Email: hoang_geol@hotmail.com typical intracontinental rift zone (Tran and Tran, 2008), is located between two large fault systems in northwestern Vietnam, including the Red River Shear zone (RRSZ) to the north and the Song Ma (Ma River) in 
Nguyen Hoang, et al./Vietnam Journal of Earth Sciences 38 (2016)

the west (Figure 1a) The Song Da rift zone consists of a series of structural zones such as Son La, Song Da, Ninh Binh and part of Thanh Hoa in the Vietnam tectonic map of Dovjikov et al (1965). Late Permian mafic, ultramafic and felsic pluton-volcanic magmatic fields are widespread in the Song
Da rift zone, including Cam Thuy, Vien Nam-Ba Vi, Kim Boi - Hoa Binh, Son La Pass, Bac Yen - Van Yen, Deo Chen, Nam Muoi, Nam So, Sin Ho and other areas (Polyakov et al., 1992, 1996; Balykin et al., 1996, 2010; Hoang et al., 2004, 2016a; Tran et al., 2011, 2015) (Figure 1a).

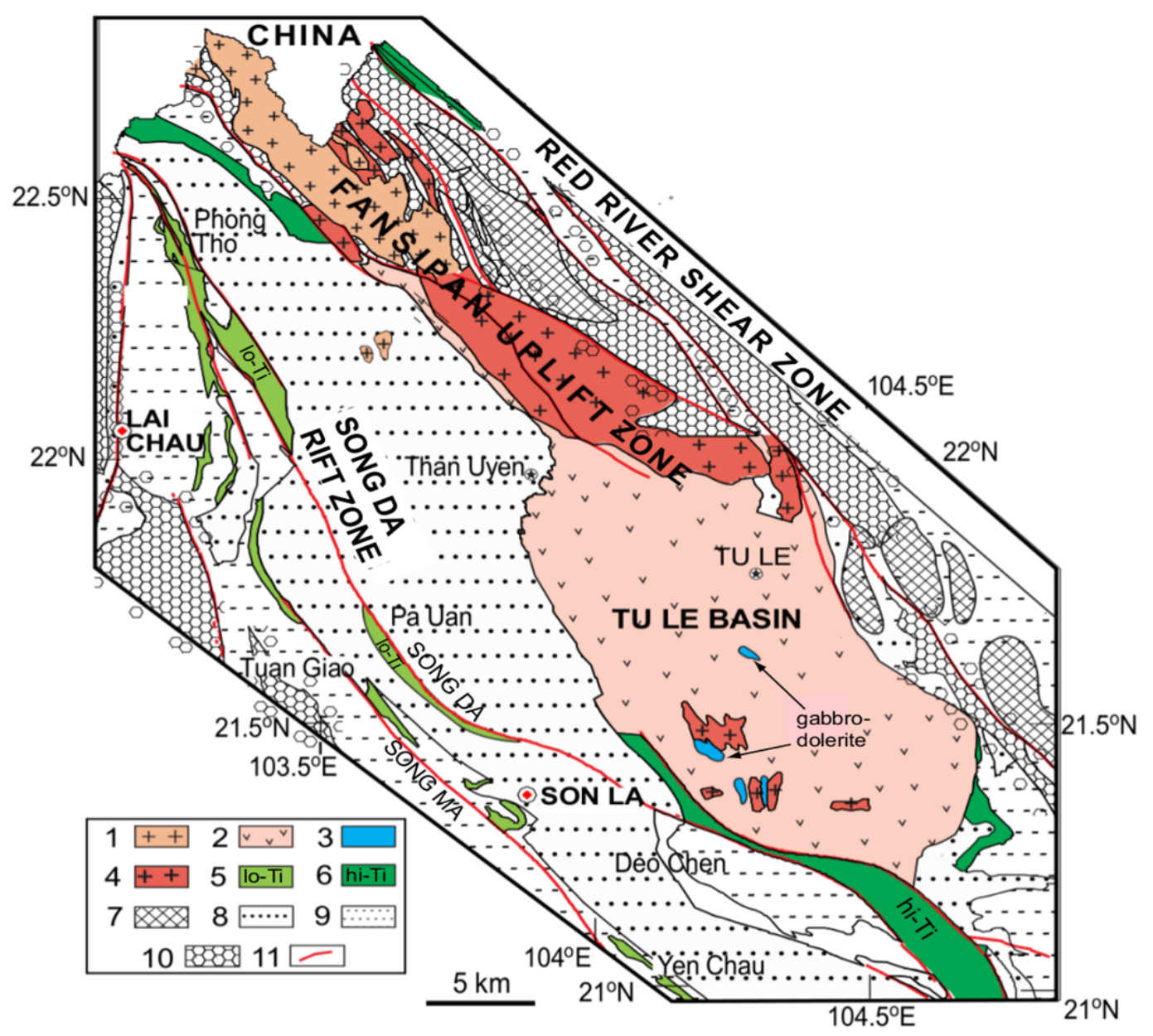

Figure 1a. Geological scheme of northwestern Viet Nam, simplified from Geological Map of Viet Nam at 1:1,500,000 (Tran and Nguyen, 1988) showing distribution areas of Song Da Permian magmatic rocks and major tectonic structures in NW Viet Nam. (1) Cenozoic granite; (2) Fan Si Pan Permian granite (Fan Si Pan uplift); (3) gabbro and dolerite; (4) Permian rhyolite and trachyte-dacite; (5) low-Ti basalt, picrite; (6) high-Ti basalt; (7) Neoproterozoic granite; (8) Mesozoic formations; (9) Paleozoic formations; (10) Pre-Cambrian metamorphic rocks (including Archean meta-granite); (11) faults 
Vietnam Journal of Earth Sciences, 38(4), 372-392

The Cam Thuy volcanic formation consists mainly of (high-Ti) basalt and andesitic basalt (basalt-andesite association) that outcrops widely in southeastern Song Ma anticlinoria, in the Cam Thuy and Tho Xuan districts (Thanh Hoa province), and scattered small centers in and around the Son La area (Son La province). This magmatic formation has long been viewed as part of the Song Da late Permian mafic - ultramafic plutonvolcanic association and termed as Cam Thuy late Permian magmatic formation (Tong Zuy Thanh and $\mathrm{Vu}$ Khuc, 2005). The magmatic rocks of Cam Thuy formation, however, have not been studied. Several authors (e.g. Tran et al., 1995, 2002, 2011, 2015; Hanski et al., 2004; Balykin et al., 2010) have suggested that the Song $\mathrm{Da}$ volcanic rocks are geochemically comparable to magmatic rocks in the Emeishan large igneous province (LIP) in SW China, which was formed by melting of deep mantle sources under the influence of a hot, deep-rooted plume (Chung and Jahn, 1995; Chung et al., 1997). The Song Da and Cam Thuy magmatic rocks are thus considered as a portion of Emeishan (E)-LIP that was extruded southeastward between the Song Ma and Red River fault zones following the India-Eurasian collision in the late Paleogene (about $30 \mathrm{Ma}$ ) (Chung et al., 1997; Wang et al., 2007; after Gilder et al., 1996).

The objectives of this study are to (1) identify the petrography and determine the elemental and $\mathrm{Sr}, \mathrm{Nd}$ and $\mathrm{Pb}$ isotopic compositions of Cam Thuy representative basalts to highlight their source region and formation parameters; (2) compare these characteristics with those of (a) Song Da and (b) Emeishan magmatic rocks that could provide evidence of their sharing a common source region.

\section{Emeishan-LIP and Song Da volcanic magmas}

\subsection{Emeishan volcanic rocks}

The Emeishan magmatic region is defined as a Large Igneous Province (LIP) for its thick magmatic layers (up to $5000 \mathrm{~m}$ ) and large distribution area (about 250,000 $\mathrm{km}^{2}$ ), occurring in Yunnan, Sichuan and Guizhou provinces, SW China. The Emeishan-LIP rests on an Early Permian limestone basement, about $1 \mathrm{~km}$ thick, possibly because of extension and subsidence tectonics in association with rifting activity of the south China plate (Xu et al., 2001). The basement was uplifted prior to the major eruption stages that formed Emeishan-LIP within a 3-millionyear span, between $253 \mathrm{Ma}$ and $250 \mathrm{Ma}(\mathrm{Xu}$ et al, 2001, 2004). Emeishan basalts are generally classified as low- and high-Ti basalt types. The low-Ti basalt is characterized by low $\mathrm{Ti} / \mathrm{Y}(<500)$ ratio, low total $\mathrm{FeO}(<12$ wt $\%)$, high $\mathrm{SiO}_{2}(48-53 \mathrm{wt} \%)$ and high $\mathrm{Mg} \#$ (52-64); whereas the high-Ti basalt type has high Ti/Y (>500) ratio, high $\mathrm{FeO}(>12.7-16.4$ wt $\%)$, low $\mathrm{SiO}_{2}(45-50 \mathrm{wt} \%)$ and high $\mathrm{Mg \#}$ (51-61) (Xu et al., 2001).

\subsection{Mafic, ultramafic magmatic rocks in the Song Da rift zone}

Permian mafic and ultramafic magmatic rocks are distributed widely in the Song $\mathrm{Da}$ rift zone. On the basis of their geochemical characteristics, the mafic and ultramafic Song Da magmatic rocks may be classified into four associations belonging to high- and lowTi magmatic types. The high-Ti andesitebasalt association outcrops in the Cam Thuy and Son La areas; the high-Ti picrite-basaltandesite association occurs in the Nam So area, and the trachyandesite, trachydacite and trachybasalt association appears in the Doi 
$\mathrm{Bu}$, Vien Nam and Nam Muoi areas. The lowTi rock type, including mafic and ultramafic volcanic rocks belongs to the picrite (komatiite?)-basalt association in the Nam Muoi, Pa Uon and Deo Chen areas (Fig. 1a) (Polyakov et al, 1991; Balykin et al, 1996, 2010; Chung et al, 1997; Tran Trong Hoa et al., 1998, 2004, 2008, 2015; Hanski et al., 2004; Nguyen Hoang et al., 2004, 2016a).

Low $\mathrm{MgO}$, high $\mathrm{FeO}, \mathrm{CaO}$ and $\mathrm{Na}_{2} \mathrm{O}$ (mafic components) contents in the high-Ti basalt in the Song Da rift zone, together with oceanic island basalt (OIB)-like trace elemental (and rare earth element) and $\mathrm{Sr}, \mathrm{Nd}$ isotopic characteristics, suggest that the highTi basalt is derived from an enriched and fertile (asthenosphere) mantle source (Hofmann, 1997; Nguyen Hoang et al., 2016a). The low-Ti basalt type, in contrast, shows high $\mathrm{MgO}$, low in the mafic component, and various trace element compositions, which reflect various geochemical features including those of island arc, mid-oceanic ridge basalt (N-MORB) and oceanic island basalt (OIB)-like type. The initial $(255 \mathrm{Ma}) \mathrm{Sr}$ and $\varepsilon_{\mathrm{Nd}}$ of the low-Ti volcanic rocks are highly variable, most certainly produced from highly heterogeneous lithospheric mantle source. In general, the Sr$\mathrm{Nd}$ isotopic compositions of Song Da Permian magmatic rocks are anomalously enriched, suggesting the melt may have interacted with crustal materials (Nguyen Hoang et al., 2016a).

\subsection{Formation ages of Emeishan and Song Da magmatism}

The radiometric age range of Song Da magmatism is controversial. Age dates obtained for Song Da magmas range from 257 $\pm 24 \mathrm{Ma}$ by Rb-Sr (Polyakov et al., 1996), of $258.5 \pm 1$ by ${ }^{40} \mathrm{Ar} /{ }^{39} \mathrm{Ar}$ (Tran Trong Hoa et al., 2008), and $270 \pm 21 \mathrm{Ma}$ by Re-Os (for 12 komatiite samples) (Hanski et al., 2004), closely matching those of Emeishan basalts (Lo et al., 2002; Zhou et al., 2002) which suggest the bulk of activity occurred between c. 251 and 259 Ma. Balykin et al. (1996) reported $\mathrm{Rb}$-Sr ages of $257 \pm 7.2 \mathrm{Ma}$ for Song Da komatiitic clinopyroxene separates from the northwestern side of the rift zone. More recently, Tang et al. (2015) reported U-Pb ages of $256.2 \pm 1.4 \mathrm{Ma}$ for zircons from a volcanic sequence in Binchuan, southern part of Emeishan LIP, and of $258.5 \pm 3.5 \mathrm{Ma}$ for a Baimazhai picrite, northwest of Jinping, immediate northern tip of the Song Da volcanic zone. Therefore, ages between 255 and $258 \mathrm{Ma}$ (late Permian) are currently taken for mafic and ultramafic magmatic rocks in the Song Da rift zone and several other nearby regional magmatic formations (e.g., Tran Trong Hoa et al., 2015; Usuki et al., 2015; Nguyen Hoang et al., 2016b).

Until recently, no reliable age has been determined for the Cam Thuy volcanic formation. While expecting new radiometric ages, we temporarily adopt ages of 258-255 Ma for Cam Thuy formation for the following reasons: (1) its geological proximity to the Song $\mathrm{Da}$ magmatic rift zone, (2) high concentration of late Permian age (258 to 255 Ma) of magmatic formations in NW Vietnam (e.g. Tang Q. et al., 2015; Tran Trong Hoa et al., 2015; Usuki et al., 2015), and (3) the availability of stratigraphic correlation-based late Permian $\left(\mathrm{P}_{3}\right)$ age for Cam Thuy volcanic formation (Tong Zuy Thanh and $\mathrm{Vu}$ Khuc, 2005 , and references therein).

\section{Sampling and analytical procedures}

Basalt sampling was conducted in the Lam Son area (Tho Xuan district, Thanh Hoa province), where massive basaltic lavas occur as large blocks along the Ho Chi Minh trail (Figs.1b-c; 2a-d), with thickness up to $600 \mathrm{~m}$ (Le Duy Bach et al., 1995). Layers of pyroclastic flows associated with the basaltic volcanism are exposed widely along the road 
Vietnam Journal of Earth Sciences, 38(4), 372-392

connecting Lam Son and Sao Vang airport (Tho Xuan town) (Fig. 1b, 2c-d). The pyroclastic products include welded, mediumgrained tuff, intercalated with layers of coarse- to fine grained volcanic ash (Fig. 2d). Individual thickness varies from a few centimeters to approximately $30 \mathrm{~cm}$, making the total visible thickness of the pyroclastic flows reach about $50 \mathrm{~m}$ (Fig. 2c-d). Samples were processed for microscopic study (Figs. 3a-f) and selection for geochemical and isotopic composition analysis.

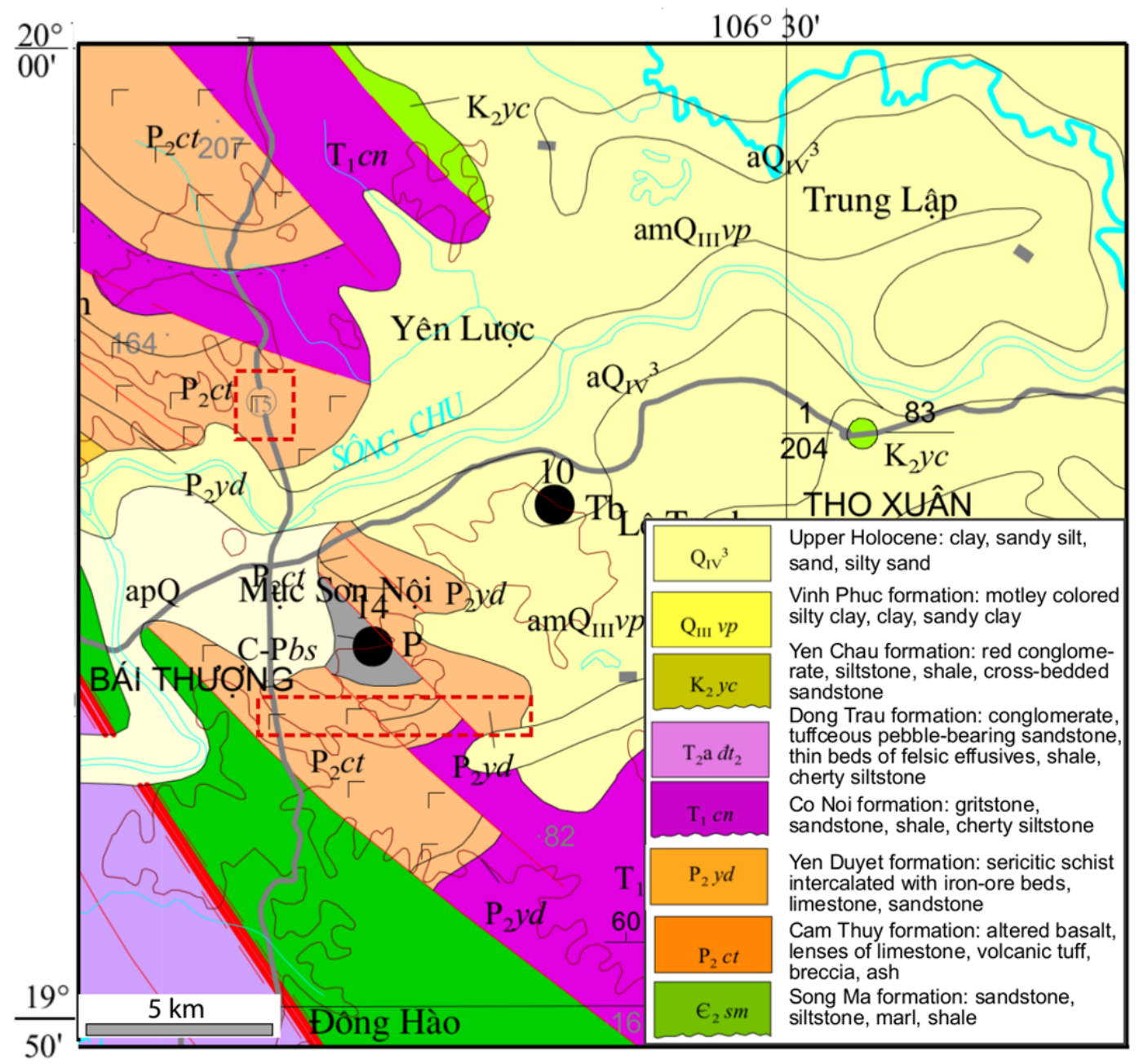

Figure 1. (b) Distribution scheme of Cam Thuy late Permian basalts in the Lam Son area (Tho Xuan, Thanh Hoa province), showing sampling sites (dashed rectangles). Simplified from 1:200,000 Geological Map of Viet Nam (after Le and Dang, 1995)

The major element compositions were acquired from fused glass beads made by mixture of sample and lithium tetraborate $\left(\mathrm{Li}_{2} \mathrm{~B}_{4} \mathrm{O}_{7}\right)$ using a Bruker Pioneer X-Ray
Fluorescence (XRF) analyzer at the Institute of Geological Sciences (VAST). Another set of samples was prepared for analysis using a Panalytical XRF at the Geological Survey of 
Nguyen Hoang, et al./Vietnam Journal of Earth Sciences 38 (2016)

Japan (GSJ) for comparison. A set of 12 GSJ geological standards were used as external data calibration and accuracy evaluation.

The trace element and rare earth element compositions were acquired at the Geological
Survey of Japan using an Agilent 8800 ICP-MS following procedures described in Ishizuka et al. (2003). The analytical accuracy as estimated from repeated measurements of GSJ standards is $\pm 1 \%$ to $\pm 6 \%$ (for $\mathrm{Nd}$ and $\mathrm{Nb}$ ).

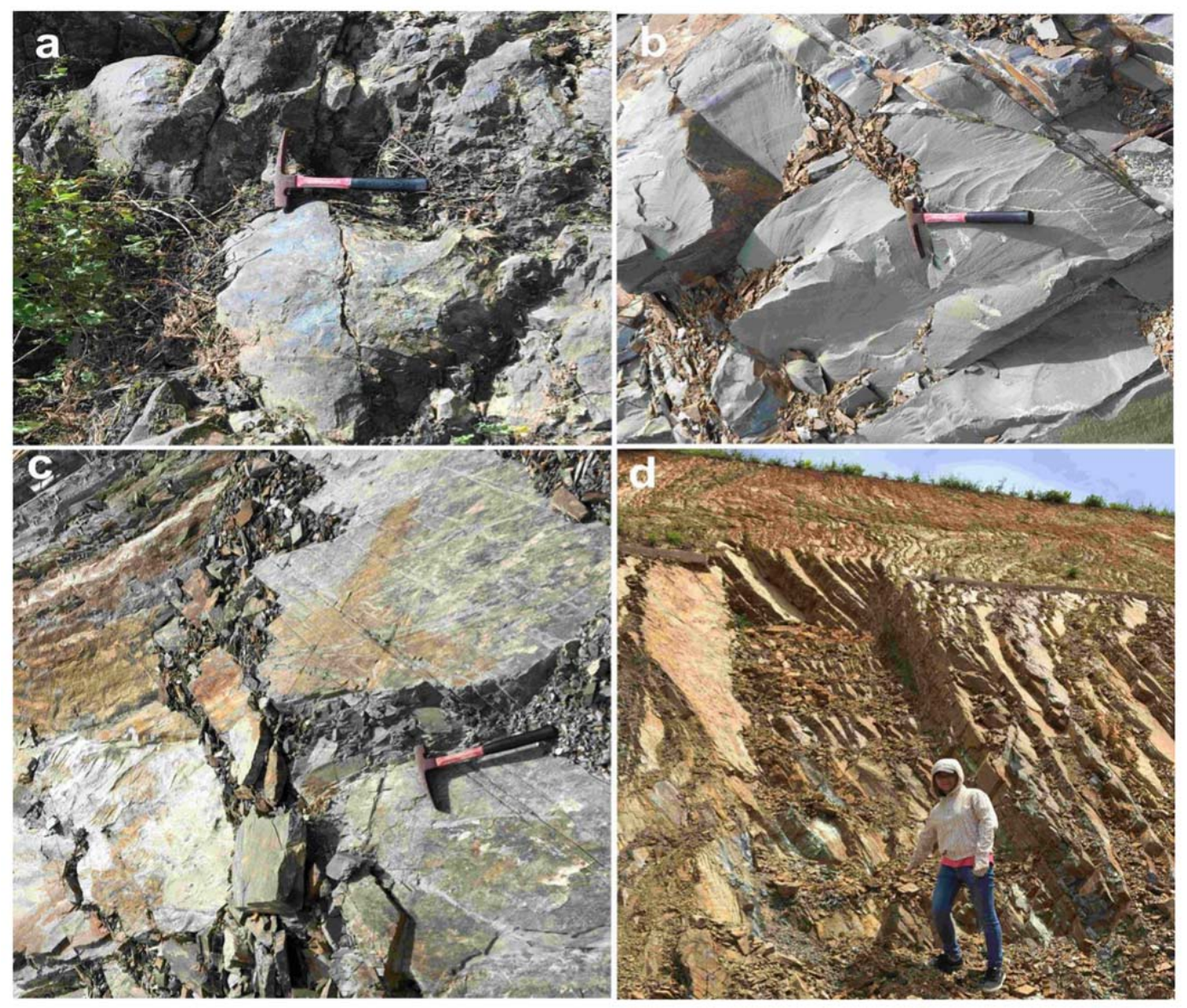

Figure 2. Outcrops Cam Thuy basalts in the Lam Son area, Tho Xuan, Thanh Hoa province: a- b: massive basaltic lava flows; c-d: volcanic pyroclastic layers

$\mathrm{Sr}, \mathrm{Nd}$ and $\mathrm{Pb}$ isotopic ratios were measured at the GSJ using a VG-54 thermal ionization mass spectrometry (TIMS, GSJ) and at the University of the Ryukyus (Okinawa, Japan) using a Neptune multi-collector (MC)-ICP-MS. The element extraction chemistry was performed at the Geological Survey of Japan. The extraction procedures and TIMS Sr, Nd, $\mathrm{Pb}$ isotopic running parameters and analytical accuracy were described in Hoang and Uto (2006) and Nguyen Hoang et al. (2013). The data are shown in Table 1. 
Vietnam Journal of Earth Sciences, 38(4), 372-392
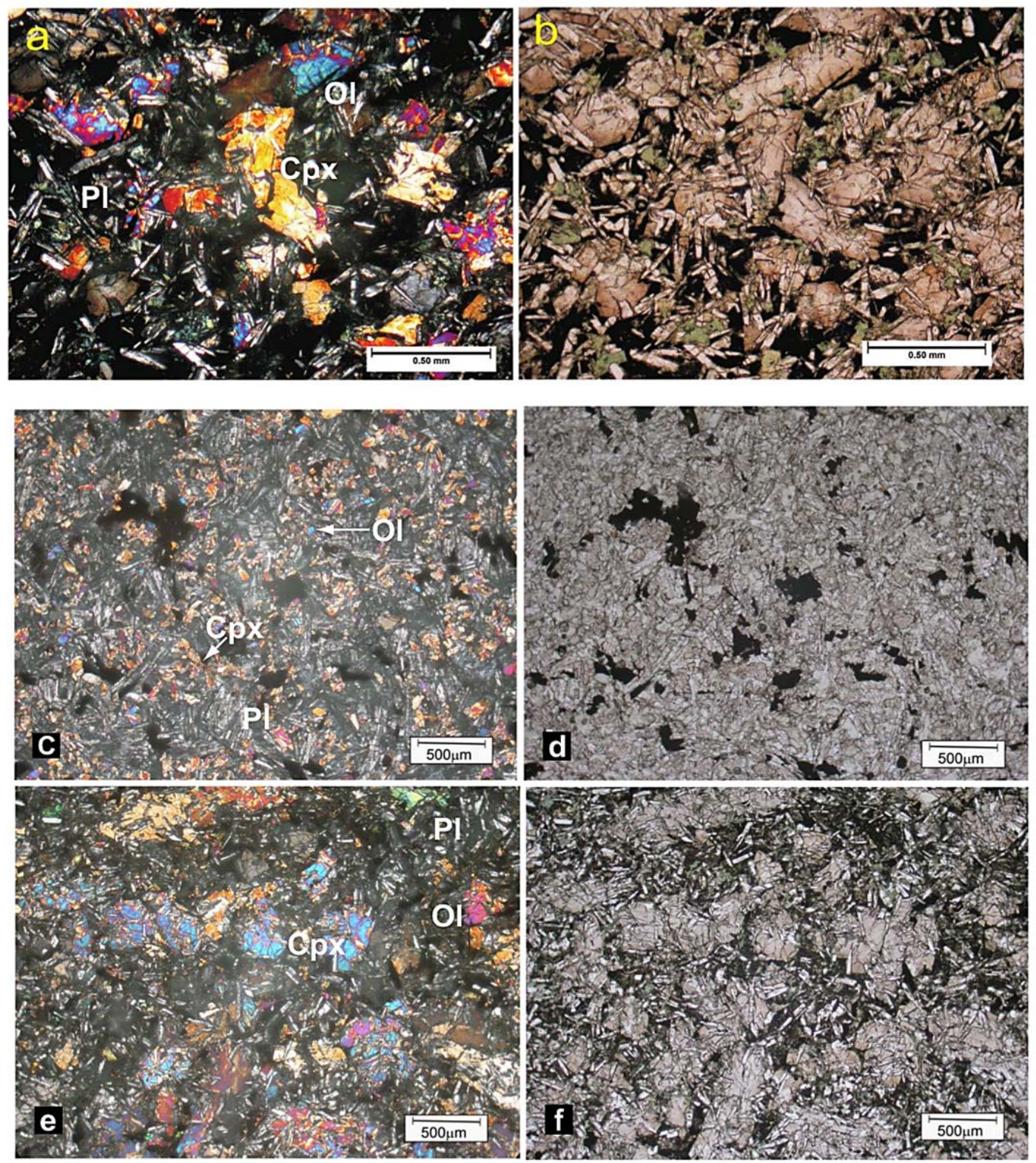

Figure 3. a, b: phyric olivine basalt (sample 040213/1) with subhedral olivine phenocrysts and needle-shaped plagioclase microlitic groundmass; c, d: aphyric alkaline basalt (sample 040213/6) containing microlites of olivine, clinopyroxene and plagioclase in the groundmass; e, f: phyric olivine basalt (sample 040213/8) with doleritic texture on the clinopyroxene and plagioclase microlitic groundmass; a, c, e: nichol (+); b, d, f: nichol (-); ruler is $0.5 \mathrm{~mm}$ 
Nguyen Hoang, et al./Vietnam Journal of Earth Sciences 38 (2016)

Table 1. Major, trace element and $\mathrm{Sr}-\mathrm{Nd}-\mathrm{Pb}$ isotopic compositions of the Cam Thuy late Permian basalts in the Lam Son area (Tho Xuan District, Thanh Hoa Province)

\begin{tabular}{|c|c|c|c|c|c|c|c|c|c|}
\hline Sample & 040213-1 & 040213-2 & 040213-3 & 040213-4 & 040213-5 & 040213-6 & $040213-7 \mathrm{~A}$ & 040213-7B & 040213-8 \\
\hline Rock type & $\begin{array}{c}\text { olivine } \\
\text { basalt }\end{array}$ & $\begin{array}{c}\text { olivine } \\
\text { basalt }\end{array}$ & $\begin{array}{c}\text { olivine } \\
\text { basalt }\end{array}$ & $\begin{array}{c}\text { olivine } \\
\text { basalt }\end{array}$ & $\begin{array}{c}\text { olivine } \\
\text { basalt }\end{array}$ & $\begin{array}{c}\text { alkaline } \\
\text { basalt }\end{array}$ & $\begin{array}{c}\text { olivine } \\
\text { basalt }\end{array}$ & $\begin{array}{c}\text { olivine } \\
\text { basalt }\end{array}$ & $\begin{array}{c}\text { tholeiitic } \\
\text { basalt }\end{array}$ \\
\hline$\overline{\mathrm{SiO}_{2}}$ & 47.70 & $\overline{47.92}$ & $\overline{48.84}$ & 48.33 & $\overline{48.58}$ & 47.99 & $\overline{48.64}$ & 49.89 & 50.33 \\
\hline $\mathrm{TiO}_{2}$ & 2.81 & 2.68 & 2.74 & 2.70 & 2.70 & 2.67 & 2.69 & 2.61 & 2.66 \\
\hline $\mathrm{Al}_{2} \mathrm{O}_{3}$ & 15.92 & 15.31 & 14.91 & 14.76 & 15.04 & 15.36 & 15.31 & 14.92 & 15.16 \\
\hline $\mathrm{FeO}^{*}$ & 12.43 & 12.98 & 12.86 & 13.26 & 12.76 & 12.70 & 12.41 & 12.14 & 11.87 \\
\hline $\mathrm{MnO}$ & 0.19 & 0.20 & 0.22 & 0.20 & 0.19 & 0.22 & 0.20 & 0.20 & 0.19 \\
\hline $\mathrm{MgO}$ & 6.17 & 5.78 & 5.63 & 6.00 & 6.09 & 5.63 & 6.31 & 6.15 & 5.65 \\
\hline $\mathrm{CaO}$ & 11.50 & 11.60 & 11.20 & 11.26 & 11.47 & 10.95 & 11.63 & 11.17 & 11.16 \\
\hline $\mathrm{Na}_{2} \mathrm{O}$ & 1.76 & 2.31 & 2.41 & 2.27 & 2.31 & 3.62 & 1.70 & 1.73 & 1.93 \\
\hline $\mathrm{K}_{2} \mathrm{O}$ & 1.12 & 0.83 & 0.79 & 0.82 & 0.49 & 0.48 & 0.72 & 0.81 & 0.65 \\
\hline $\mathrm{P}_{2} \mathrm{O}_{5}$ & 0.41 & 0.39 & 0.40 & 0.40 & 0.40 & 0.39 & 0.39 & 0.38 & 0.39 \\
\hline $\mathrm{Mg \#}$ & 52.5 & 49.8 & 49.4 & 50.2 & 51.5 & 49.7 & 53.1 & 53.0 & 51.5 \\
\hline $\mathrm{Ti}(\mathrm{ppm})$ & 16830 & 16065 & 16419 & 16190 & 16178 & 16029 & 16137 & 15662 & 15949 \\
\hline $\mathrm{K}(\mathrm{ppm})$ & 9306 & 6909 & 6555 & 6776 & 4029 & 3967 & 5947 & 6742 & 5361 \\
\hline $\mathrm{Na}_{2} \mathrm{O}+\mathrm{K}_{2} \mathrm{O}$ & 2.88 & 3.14 & 3.20 & 3.09 & 2.79 & 4.10 & 2.42 & 2.54 & 2.58 \\
\hline \multicolumn{10}{|l|}{ CIPW } \\
\hline Quartz & & & & & & & & & 2.05 \\
\hline Orthoclase & 6.62 & 4.92 & 4.67 & 4.82 & 2.87 & 2.82 & 4.23 & 4.80 & 3.82 \\
\hline Albite & 14.85 & 19.53 & 20.35 & 19.25 & 19.54 & 24.38 & 14.39 & 14.61 & 16.34 \\
\hline Anorthite & 32.24 & 28.95 & 27.57 & 27.64 & 29.23 & 24.26 & 32.02 & 30.56 & 30.80 \\
\hline Nepheline & & & & & & 3.38 & & & \\
\hline Diopside & 18.45 & 21.81 & 21.22 & 21.41 & 20.93 & 23.02 & 19.26 & 18.64 & 18.38 \\
\hline Hypersthene & 12.60 & 5.99 & 11.90 & 10.31 & 13.91 & & 21.96 & 24.38 & 22.67 \\
\hline Olivine & 8.98 & 12.83 & 8.17 & 10.53 & 7.50 & 16.18 & 2.13 & & \\
\hline \multicolumn{10}{|l|}{ Magnetite } \\
\hline Ilmenite & 5.33 & 5.09 & 5.20 & 5.13 & 5.12 & 5.08 & 5.11 & 4.96 & 5.05 \\
\hline Apatite & 0.94 & 0.90 & 0.94 & 0.93 & 0.92 & 0.91 & 0.91 & 0.89 & 0.90 \\
\hline
\end{tabular}

Table 1. (continued)

\begin{tabular}{|c|c|c|c|c|c|c|c|c|c|}
\hline Sample & 040213-1 & 040213-3 & $040213-5$ & 040213-6 & 040213-7A & CT150616-C & CT150616-G & CT150616-J & CT150616-L \\
\hline Rock type & $\begin{array}{c}\text { olivine } \\
\text { basalt }\end{array}$ & $\begin{array}{c}\text { olivine } \\
\text { basalt }\end{array}$ & $\begin{array}{c}\text { olivine } \\
\text { basalt }\end{array}$ & $\begin{array}{c}\text { alkaline } \\
\text { basalt }\end{array}$ & $\begin{array}{c}\text { alkaline } \\
\text { basalt }\end{array}$ & $\begin{array}{c}\text { olivine } \\
\text { basalt }\end{array}$ & tholeiite & $\begin{array}{c}\text { alkaline } \\
\text { basalt }\end{array}$ & $\begin{array}{c}\text { olivine } \\
\text { basalt }\end{array}$ \\
\hline$\overline{\mathrm{Rb}}$ & 23.26 & 22.66 & $\overline{9.72}$ & $\overline{9.43}$ & 12.95 & 21.01 & 10.26 & 14.67 & 6.61 \\
\hline $\mathrm{Sr}$ & 322.14 & 370.49 & 292.18 & 739.52 & 360.62 & 327.80 & 256.51 & 450.26 & 517.99 \\
\hline $\mathrm{Y}$ & 33.23 & 31.26 & 32.61 & 30.99 & 32.54 & 27.29 & 29.16 & 28.91 & 33.58 \\
\hline $\mathrm{Zr}$ & 177.22 & 161.86 & 176.63 & 167.93 & 172.07 & 155.53 & 164.68 & 185.71 & 191.21 \\
\hline $\mathrm{Nb}$ & 22.94 & 20.84 & 21.76 & 21.32 & 22.18 & 18.95 & 19.10 & 27.98 & 28.38 \\
\hline Cs & 0.57 & 0.86 & 1.17 & 0.57 & 0.53 & 0.50 & 0.08 & 0.26 & 0.11 \\
\hline $\mathrm{Ba}$ & 261.04 & 302.32 & 130.16 & 149.33 & 153.96 & 285.94 & 124.37 & 601.53 & 530.67 \\
\hline $\mathrm{La}$ & 25.09 & 25.26 & 27.15 & 23.88 & 26.52 & 20.80 & 25.04 & 31.95 & 34.49 \\
\hline $\mathrm{Ce}$ & 58.15 & 54.61 & 57.73 & 53.57 & 58.42 & 48.15 & 53.99 & 76.70 & 82.07 \\
\hline $\operatorname{Pr}$ & 7.10 & 6.64 & 6.88 & 6.65 & 7.09 & 5.81 & 6.75 & 9.75 & 10.18 \\
\hline $\mathrm{Nd}$ & 31.54 & 29.58 & 31.07 & 29.51 & 31.82 & 25.28 & 30.13 & 43.50 & 45.50 \\
\hline $\mathrm{Sm}$ & 7.01 & 6.70 & 6.83 & 6.55 & 7.02 & 5.73 & 6.66 & 8.72 & 9.32 \\
\hline $\mathrm{Eu}$ & 2.40 & 2.25 & 2.22 & 2.31 & 2.33 & 2.11 & 2.44 & 3.43 & 3.74 \\
\hline $\mathrm{Gd}$ & 6.94 & 6.55 & 6.55 & 6.42 & 6.90 & 5.60 & 6.45 & 7.78 & 8.66 \\
\hline $\mathrm{Tb}$ & 1.11 & 1.04 & 1.06 & 1.04 & 1.09 & 0.89 & 1.00 & 1.12 & 1.24 \\
\hline Dy & 6.26 & 5.73 & 6.01 & 5.86 & 6.19 & 5.11 & 5.68 & 6.17 & 6.55 \\
\hline Ho & 1.21 & 1.15 & 1.19 & 1.16 & 1.23 & 1.00 & 1.08 & 1.12 & 1.25 \\
\hline $\mathrm{Er}$ & 3.31 & 3.19 & 3.08 & 3.15 & 3.12 & 2.69 & 2.86 & 2.93 & 3.25 \\
\hline $\mathrm{Tm}$ & 0.47 & 0.44 & 0.45 & 0.44 & 0.44 & 0.40 & 0.40 & 0.40 & 0.47 \\
\hline$\underline{\mathrm{Yb}}$ & 2.90 & 2.71 & 2.77 & 2.68 & 2.81 & 2.35 & 2.43 & 2.51 & 2.65 \\
\hline
\end{tabular}


Vietnam Journal of Earth Sciences, 38(4), 372-392

\begin{tabular}{lrrrrrrrrr}
\hline $\mathrm{Lu}$ & 0.40 & 0.41 & 0.39 & 0.39 & 0.39 & 0.33 & 0.35 & 0.36 & 0.39 \\
$\mathrm{Hf}$ & 4.82 & 4.38 & 4.55 & 4.46 & 4.64 & 4.12 & 4.44 & 4.94 & 5.01 \\
$\mathrm{Ta}$ & 1.79 & 1.61 & 1.66 & 1.64 & 1.69 & 1.46 & 1.47 & 2.20 & 2.19 \\
$\mathrm{~Pb}$ & 38.67 & 2.86 & 2.89 & 3.05 & 3.27 & 3.38 & 2.92 & 4.64 & 2.95 \\
$\mathrm{Th}$ & 3.64 & 3.35 & 3.39 & 3.38 & 3.45 & 2.80 & 2.79 & 3.47 & 3.54 \\
$\mathrm{U}$ & 0.90 & 0.74 & 0.80 & 0.70 & 0.90 & 0.55 & 0.63 & 0.25 & 0.87 \\
$\mathrm{~V}$ & 324.98 & 326.07 & 330.74 & 336.07 & 357.86 & 337.43 & 321.15 & 406.91 & 401.09 \\
$\mathrm{Cr}$ & 150.18 & 148.35 & 161.59 & 152.94 & 157.01 & 261.50 & 208.18 & 95.69 & 102.91 \\
$\mathrm{Ni}$ & 95.44 & 92.71 & 95.19 & 91.88 & 92.33 & 147.54 & 110.99 & 75.38 & 80.74 \\
\hline
\end{tabular}

Table 1. (continued)

\begin{tabular}{|c|c|c|c|c|c|c|c|c|c|}
\hline ample & 40213-1 & $040213-3$ & 040213-5 & $040213-6$ & $-040213-7 \mathrm{~A}$ & $=$ & T150616-G & CT150616-J & CT150616-L \\
\hline Rock type & $\begin{array}{c}\text { olivine } \\
\text { basalt }\end{array}$ & $\begin{array}{c}\text { olivine } \\
\text { basalt }\end{array}$ & $\begin{array}{c}\text { olivine } \\
\text { basalt }\end{array}$ & $\begin{array}{c}\text { alkaline } \\
\text { basalt }\end{array}$ & $\begin{array}{c}\text { alkaline } \\
\text { basalt }\end{array}$ & $\begin{array}{c}\text { olivine } \\
\text { basalt }\end{array}$ & tholeiite & $\begin{array}{c}\text { alkaline } \\
\text { basalt }\end{array}$ & $\begin{array}{c}\text { alkaline } \\
\text { basalt }\end{array}$ \\
\hline${ }^{87} \mathrm{Rb} /{ }^{86} \mathrm{Sr}$ & 0.228 & $\overline{0.202}$ & 0.107 & 0.042 & 0.115 & 0.179 & 0.112 & 0.091 & 0.0356 \\
\hline${ }^{87} \mathrm{Sr} /{ }^{86} \mathrm{Sr}_{\mathrm{m}}$ & 0.706105 & 0.706269 & 0.705815 & 0.705986 & 0.705926 & 0.705989 & 0.706274 & .705655 & 0.705462 \\
\hline${ }^{87} \mathrm{Sr} /{ }^{86} \mathrm{Sr}_{(255 \mathrm{Ma})}$ & 0.705279 & 0.705536 & 0.705429 & 0.705836 & 0.705507 & 0.705340 & 0.705869 & 0.705325 & 0.7053327 \\
\hline${ }^{147} \mathrm{Sm} /{ }^{144} \mathrm{Nd}_{\mathrm{m}}$ & 0.142 & 0.140 & 0.136 & 0.137 & 0.136 & 0.140 & 0.137 & 0.124 & 0.126 \\
\hline${ }^{143} \mathrm{Nd} /{ }^{144} \mathrm{Nd}_{\mathrm{m}}$ & 0.512568 & .512578 & .512464 & 0.512570 & 0.512573 & 0.512615 & 512598 & 512572 & 512584 \\
\hline$\varepsilon_{\mathrm{Nd}}$ & -1.36 & -1.17 & -3.40 & -1.32 & -1.27 & -0.45 & -0.78 & -1.29 & -1.05 \\
\hline $\mathrm{d}(255 \mathrm{Ma})$ & 0.43 & 0.67 & -1.42 & 0.61 & 0.69 & 1.40 & 1.17 & 1.08 & 1.23 \\
\hline${ }^{3} \mathrm{Nd} /{ }^{144} \mathrm{Nc}$ & 0.512332 & 0.512345 & 0.512237 & 0.512341 & 0.512345 & 0.512382 & 0.512370 & 0.512365 & 0.512373 \\
\hline $\mathrm{T}_{(\mathrm{DM})}$ & $1.23 \mathrm{E}+09$ & $18 \mathrm{E}+09$ & $.34 \mathrm{E}+09$ & $1.16 \mathrm{E}+09$ & $1.14 \mathrm{E}+09$ & $1.1 \mathrm{E}+09$ & $1.1 \mathrm{E}+09$ & $9.8 \mathrm{E}+08$ & $9.9 \mathrm{E}+08$ \\
\hline (ppm) & 0.90 & 0.74 & 0.80 & 0.70 & 0.90 & 0.548 & 0.627 & 0.247 & 0.865 \\
\hline $\mathrm{h}(\mathrm{ppm})$ & 3.65 & 3.35 & 3.39 & 3.38 & 3.45 & 2.802 & 2.794 & 3.470 & 3.542 \\
\hline (ppm) & 5.3 & 4.8 & 3.6 & 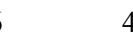 & 1.2 & 3.382 & 2.916 & 4.638 & 2.945 \\
\hline${ }^{238} \mathrm{U} /{ }^{204} \mathrm{~Pb}$ & 1.39 & 15.72 & 16.83 & 13.94 & 16.68 & 9.847 & 13.061 & 3.240 & 17.848 \\
\hline${ }^{235} \mathrm{U} /{ }^{204} \mathrm{~Pb}$ & 0.01 & 0.12 & 0.12 & 0.10 & 0.12 & 0.072 & 0.096 & 0.024 & 0.131 \\
\hline${ }^{232} \mathrm{Th} /{ }^{204} \mathrm{~Pb}$ & 5.84 & 73.70 & 73.56 & 69.56 & 66.26 & 52.027 & 60.165 & 46.976 & 75.520 \\
\hline${ }^{206} \mathrm{~Pb} /{ }^{204} \mathrm{~Pb}_{\mathrm{m}}$ & 18.309 & 19.291 & 19.439 & 19.348 & 19.436 & 19.189 & 19.271 & 18.674 & 19.282 \\
\hline${ }^{7} \mathrm{~Pb} /{ }^{204} \mathrm{~Pb}_{\mathrm{m}}$ & 15.606 & 15.647 & 15.638 & 15.626 & 15.647 & 15.632 & 15.631 & 15.594 & 15.622 \\
\hline${ }^{208} \mathrm{~Pb} /{ }^{204} \mathrm{~Pb}_{\mathrm{m}}$ & 38.357 & 39.725 & 39.813 & 39.787 & 39.791 & 39.583 & 39.625 & 39.424 & 39.681 \\
\hline${ }^{006} \mathrm{~Pb} /{ }^{204} \mathrm{~Pb}_{(255 \mathrm{~N}}$ & 18.253 & 18.670 & 18.774 & 18.797 & 18.777 & 18.792 & 18.744 & 18.543 & 18.562 \\
\hline${ }^{207} \mathrm{~Pb} /{ }^{204} \mathrm{~Pb}_{(255 \mathrm{Ma})}$ & 15.603 & 15.615 & 15.604 & 15.597 & 15.613 & 15.611 & 15.604 & 15.587 & 15.585 \\
\hline${ }^{208} \mathrm{~Pb} /{ }^{204} \mathrm{~Pb}_{(255 \mathrm{Ma})}$ & 38.284 & 38.808 & 38.897 & 38.921 & 38.966 & 38.922 & 38.861 & 38.827 & 38.722 \\
\hline
\end{tabular}

\section{Analytical results}

\subsection{Petrographic characteristics}

The analyzed samples are mostly phyric olivine basalts and subsidiary alkaline basalts with major phenocrysts of olivine constituting about 3 to 5 vol.\% (Figs. 3a-f). The groundmass is intersertal, micro-doleritic, containing microlites of clinopyroxene, plagioclase, ore minerals, (rare) olivine and volcanic glass (Figs. 3c-d). Olivine phenocrysts are euhedral or subhedral, tabletor irregular shaped, with sizes ranging from 0.1 by $0.3 \mathrm{~mm}$ to 0.3 by $0.5 \mathrm{~mm}$ (Fig. 2a-c). Some alkaline basalts contain iddingsite, a product of altered olivine. Some basalts are aphyric with the groundmass comprising microlites of clinopyroxene and plagioclase (Fig. 3c-d, e-f). The welded tuff contains fragments of altered lavas cemented by volcanic ash (Figs. 4c-d). The volcanic ash is coarse- or fine grained (Fig. 2d), disoriented (Fig. 5a) or layered and oriented (Fig. 5b).

\subsection{Major element compositions}

Cam Thuy basalts (in the Lam Son area) with $\mathrm{SiO}_{2}$, varying from 47.70 to 50.33 wt.\% and total alkaline oxides $\left(\mathrm{Na}_{2} \mathrm{O}+\mathrm{K}_{2} \mathrm{O}\right)$ from 2.42 to $4.10 \mathrm{wt} \%$, are distributed in the subalkaline field, while only a few samples plot in the alkaline field (Fig. 6). This features 
Nguyen Hoang, et al./Vietnam Journal of Earth Sciences 38 (2016)

are expressed in terms of CIPW normative mineralogical compositions showing that only one sample $(040213 / 6)$ contains nepheline (Ne)-normative of 3.38 wt.\% (Table 1), and all remaining samples are subakaline rock type (containing olivine (Ol)-normative) or tholeiitic basalt, containing quartz $(\mathrm{Q})$ - and hypersthene (Hy)-normative (Table 1).

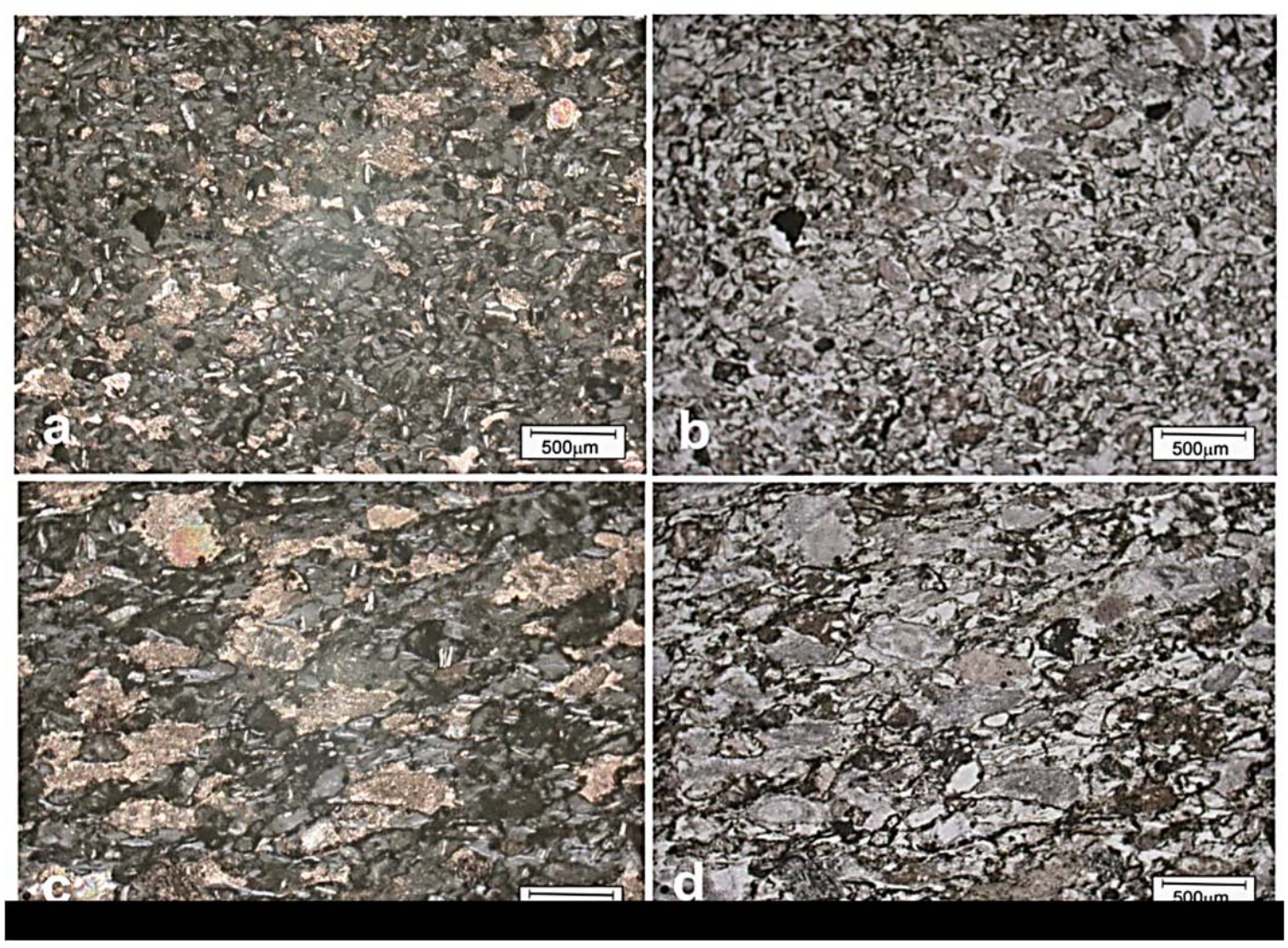

Figure 4. a-b (sample CT150616-E) and c-d (samplCT150616-F) tuff composed of basaltic fragments of variable sizes, cemented by volcanic ash; layered and oriented; fragments are partially chloritized, carbonatized and albitized lava
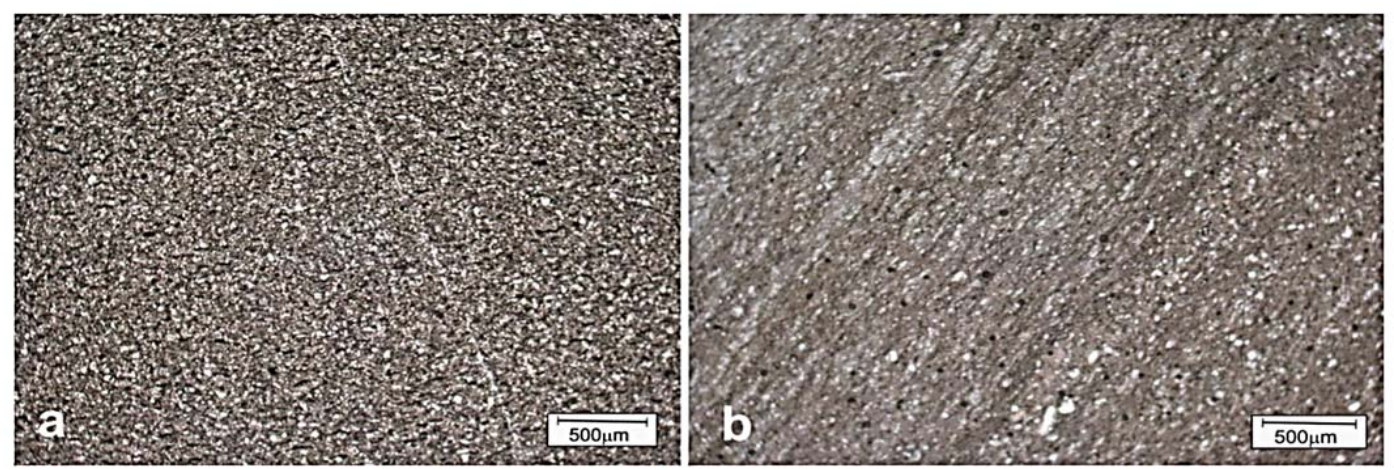

Figure 5. a: (sample CT150616-G); b: (CT150616-H) volcanic ash, coarse- or fine-grained, with or without orientation and zonation 
$\mathrm{MgO}$ contents of the Cam Thuy basalts vary from 5.8 wt.\% to 7.2 wt.\%, plotted between $\mathrm{MgO}$ values of the Song $\mathrm{Da}$ and Emeishan basalts (Fig. 7). In a similar way, except for having much higher $\mathrm{CaO}$ contents than those of the Song $\mathrm{Da}$ and
Emeishan basalts, the other oxides such as $\mathrm{SiO}_{2}, \mathrm{TiO}_{2}, \mathrm{FeOt}, \mathrm{Na}_{2} \mathrm{O}$ and $\mathrm{K}_{2} \mathrm{O}$ of the Cam Thuy basalts plot between fields of the Song $\mathrm{Da}$ and Emeishan magmatic rocks and almost overlap those of high-Ti basalt type (Fig. 7).
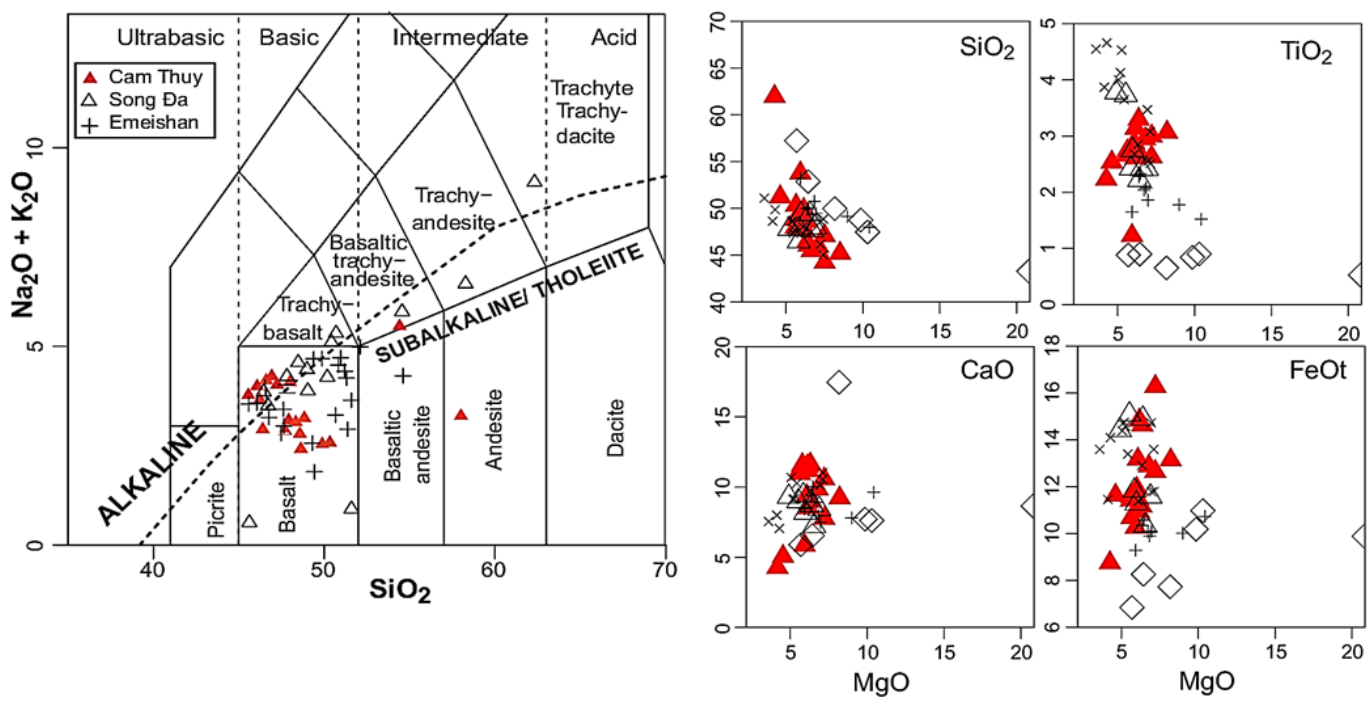

Figure 6. Basaltic TAS (total alkalis vs. $\mathrm{SiO}_{2}$ ) Figure 7. Correlation between $\mathrm{MgO}$ (wt.\%) and major classification (after Le Bas et al., 1986) showing samples silicate oxides showing Emeishan and Song Da basalts of Cam Thuy basalt (this study); Song Da basalts (Hoang with $\mathrm{CaO}$ values being lower compared with the Cam et al., 2016) and Emeishan samples (Xu et al., 2001, Thuy basalts, while $\mathrm{TiO}_{2}$ contents of Cam Thuy basalts 2004). Cam Thuy basalts mostly overlap those of Song Da being comparable to Song Da high-Ti basalts (empty high-Ti basalt series and plot between high- and low-Ti triangle) but lower than Emeishan high-Ti basalts of Emeishan magmas

(cross); symbols for Song Da low-Ti: empty diamond, Emeishan low-Ti magma: $\mathrm{x}$

\subsection{Trace element compositions}

Primitive mantle normalized trace element (Hofmann, 1988) and chondrite normalized rare earth element (Anders and Grevesse, 1989) distribution patterns of the Cam Thuy basalts are shown (Figs. 8a, 9a, respectively) along with Song Da high-Ti basalts (Son La Pass and nearby areas) (Figs. 8b, 9b) and geological standards made from volcanic rocks of various tectonic settings (Figs. 8c, 9c) for comparison (N. Hoang et al., 2016a).

The trace and rare earth element distribution patterns of Cam Thuy basalts show smooth decrease from left to right (Figs.8b, 7b), almost overlapping trace and rare earth element distribution curve of the Song Da high-Ti basalts (Figs. 8b, 9b) (data from Hoang et al., 2016a). The basalt samples both of Song $\mathrm{Da}$ and Cam Thuy show patterns, which are closely analogous to oceanic island basalts (e.g. JB-1a and BHVO2 in Figures 8c and 9c). 
Nguyen Hoang, et al./Vietnam Journal of Earth Sciences 38 (2016)
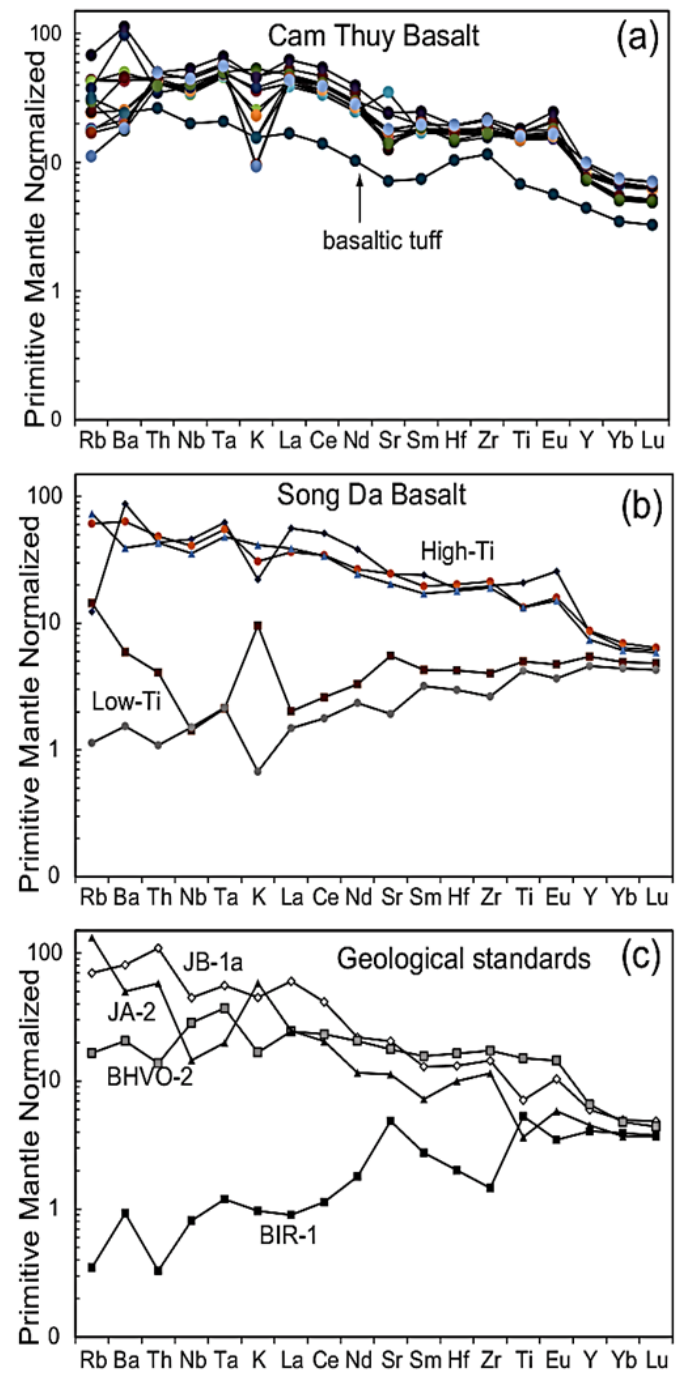

Figure 8. Primitive mantle normalized trace element patterns of Cam Thuy basalts (a) as compared to Song Da high-Ti basalts (b); geological standards (BIR-1: Indian MORB; BHVO-2: Hawaiian OIB; JB-1a: continental intraplate basalt; JA-2: arc andesite) are shown for comparison (c). Normalizing data are after Hofmann (1988). Note that the trace element distribution patterns of Cam Thuy basalts being closely analogous to Song Da high-Ti basalts are essentially oceanic island basalt-like
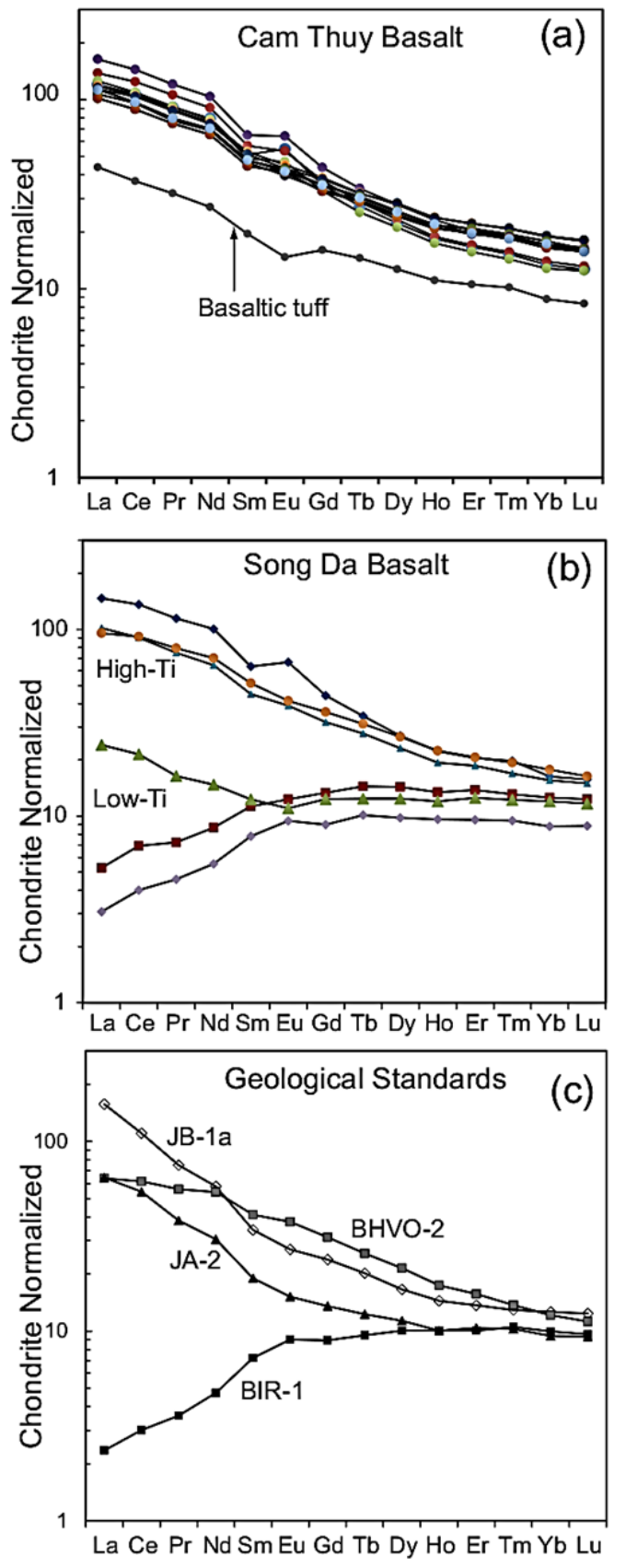

Figure 9

$\rightarrow$ Figure 9. Chondrite normalized rare earth element (after Anders and Grevesse, 1989) distribution patterns of Cam Thuy basalts (a); shown are Song Da high-Ti basalts (b) and geological standards (c) for comparison (see Figure 8 caption). The rare earth element configuration curves of Cam Thuy basalts are comparable to Song Da high-Ti basalts while vastly different from the low-Ti basalt type, having a N-MORB shape (BIR-1) 
Vietnam Journal of Earth Sciences, 38(4), 372-392

\subsection{Isotopic compositions}

The initial ${ }^{87} \mathrm{Sr} /{ }^{86} \mathrm{Sr}$ ratios of the Cam Thuy basalts calculated for $255 \mathrm{Ma}$ after Song Da basaltic eruption age (e.g. Balykin et al., 1996; Tran Trong Hoa et al., 2008, after Tang Q. et al., 2015) range from 0.70528 to 0.70584 . These initial isotopic ratios accompanied by $\varepsilon_{\mathrm{Nd}(255 \mathrm{Ma})}$ varying from 0.69 to -1.41 , are plotted between two fields of depleted mantle (DM) and enriched continental crust (Fig. 10), overlapping the field of the Song Da high-Ti basalt and covering partially that of Emeishan high-Ti basalt. The initial ${ }^{87} \mathrm{Sr} /{ }^{86} \mathrm{Sr}$ ratios of the Cam Thuy basalts are lower as compared with Emeishan basalts and much lower compared with Song Da low-Ti basalts having $255 \mathrm{Ma}$ initial ${ }^{87} \mathrm{Sr} /{ }^{86} \mathrm{Sr}$ ratios from 0.7055 to 0.7115 accompanied by $\varepsilon_{\mathrm{Nd}(255 \mathrm{Ma})}$ changing between 7.5 and -9 (Xu et al., 2001; Nguyen Hoang et al., 2004, 2016a).

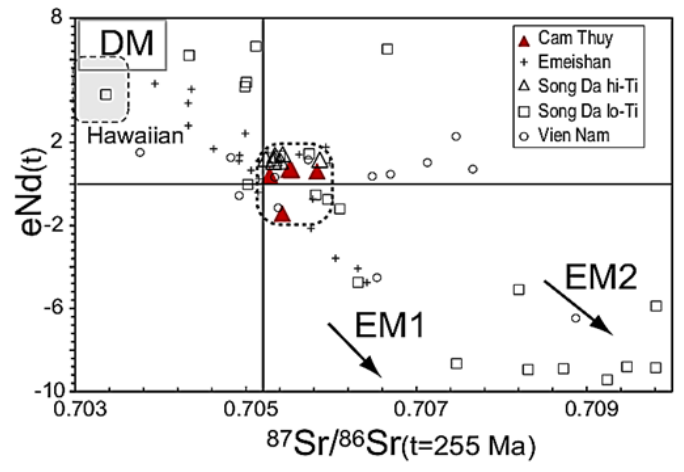

Figure 10. Plots of initial $(255 \mathrm{Ma}){ }^{87} \mathrm{Sr} /{ }^{86} \mathrm{Sr}$ isotopic ratios versus $\varepsilon_{\mathrm{Nd}(t)}$ of Cam Thuy magmatic rocks. Emeishan and Song $\mathrm{Da}$ basalts are shown for comparison. Fields of depleted mantle (DM) and enriched mantle type 1 and 2 (EM1, EM2) and Hawaiian OIB (data from Norman and Garcia, 1999) are shown for reference. Note Cam Thuy basaltic distribution field includes that of Song $\mathrm{Da}$ high-Ti basalts away from Song Da low-Ti and Emeishan magmatic rocks

The initial ${ }^{206} \mathrm{~Pb} /{ }^{204} \mathrm{~Pb}$ ratios $(255 \mathrm{Ma})$ of the Cam Thuy basalts plotted against ${ }^{207} \mathrm{~Pb} /{ }^{204} \mathrm{~Pb}_{\mathrm{i}}$ and ${ }^{208} \mathrm{~Pb} /{ }^{204} \mathrm{~Pb}_{\mathrm{i}}$ ratios are shown in Fig. 11a-b. The lead isotopic ratios plot close to the depleted mantle (DM) field (represented by Pacific MORB), overlapping the field of the Song Da high-Ti basalt and separating from the field of the low-Ti basalt. The latter, with higher ${ }^{206} \mathrm{~Pb} /{ }^{204} \mathrm{~Pb}_{\mathrm{i}},{ }^{207} \mathrm{~Pb} /{ }^{204} \mathrm{~Pb}_{\mathrm{i}}$ and ${ }^{208} \mathrm{~Pb} /{ }^{204} \mathrm{~Pb}_{\mathrm{i}}$, trend toward enriched fields.
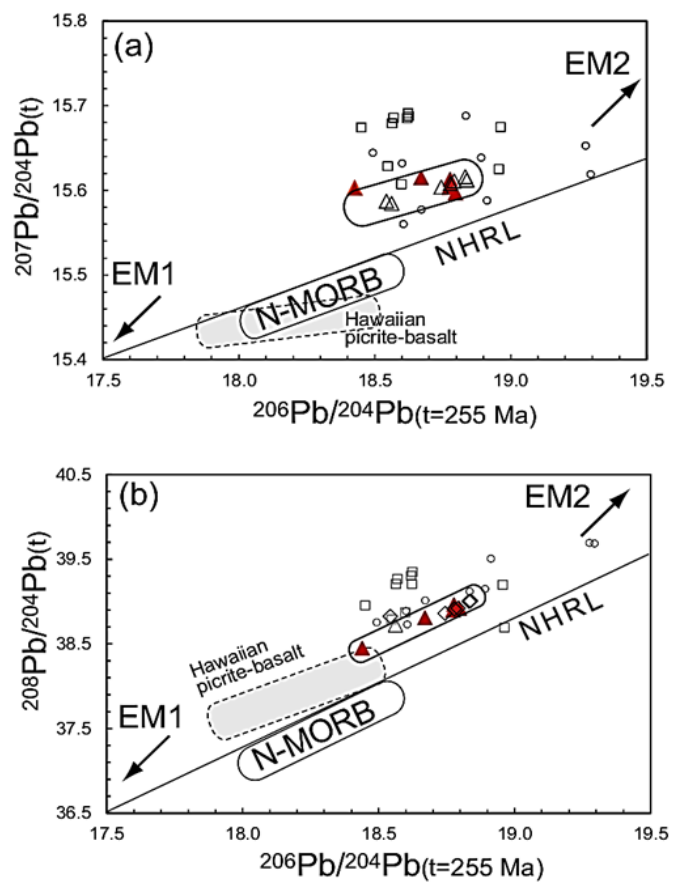

Figure 11. Plots of initial (255Ma) ${ }^{206} \mathrm{~Pb} /{ }^{204} \mathrm{~Pb}$ isotopic ratios versus (a) ${ }^{207} \mathrm{~Pb} /{ }^{204} \mathrm{~Pb}$ and (b) ${ }^{208} \mathrm{~Pb} /{ }^{204} \mathrm{~Pb}$ of Cam Thuy basalts as compared to Song Da basalts; EM2, EM1 (enriched mantle type 1 and 2), Hawaiian OIB (Norman and Garcia, 1999) and (depleted) Mid-Ocean Ridge Basalt (N-MORB) are shown for reference. Symbols as in Figure 10. Northern Hemisphere Reference Line (NHRL) illustrates enriched (above) and depleted mantle domains

\section{Discussion}

\subsection{Tectonic setting of Cam Thuy basalts}

Igneous rocks formed in different tectonic settings may be affected by in situ materials at different levels. For example, at a subduction zone, magmatic melts may be affected by oceanic crustal material, brought to the mantle by the subducting slab, in the form of hydrous 
Nguyen Hoang, et al./Vietnam Journal of Earth Sciences 38 (2016)

fluid, which appears to be one of the causes of lowering melting temperature of the mantle wedge. Using $\mathrm{Zr} / \mathrm{Y}$ plotted against $\mathrm{Zr}$ in the tectonic setting discrimination diagram (after Pearce and Norry, 1979), the Cam Thuy basalts, Emeishan and most of Song Da magmatic rocks plot in the field of intraplate magmas (Fig. 12). Some Song Da low-Ti basalts plot in the subduction field, making them different from the Cam Thuy lavas.

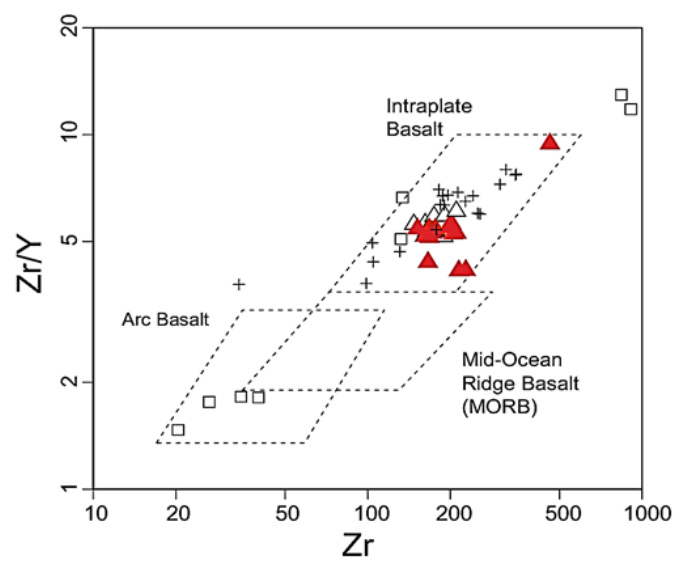

Figure 12. Tectonic discrimination diagram (after Pearce and Norry, 1979) showing Cam Thuy basalts plotting in intraplate basalt field comparable to Emeishan basalts and many of the Song Da magmatic rocks; some Song Da low-Ti basalts plot in field of island arc. Symbols as in Figure 10

Basaltic melts on the way to the surface may interact with crustal rocks. Crustal material interaction may result in increasing $\mathrm{Ba}, \mathrm{Rb}, \mathrm{Th}$, etc., contents relative to $\mathrm{Nb}, \mathrm{Ta}$, $\mathrm{Zr}, \ldots$ in basaltic melts, forming positive correlation between (for example) $\mathrm{Ba} / \mathrm{Nb}$ against $\mathrm{SiO}_{2}$ and negative correlation with $\mathrm{MgO}$ (or $\mathrm{Mg \# ).} \mathrm{Cam} \mathrm{Thuy} \mathrm{basalts} \mathrm{have} \mathrm{low}$ $\mathrm{SiO}_{2}$ and $\mathrm{K}_{2} \mathrm{O}$ contents and show oceanic island basalt-like trace element distribution patterns (Figs. 8, 9), indicating minimal crustal involvement. Besides, correlation between $\mathrm{Ti} / \mathrm{Zr}$ against $\mathrm{Ba} / \mathrm{Zr}$ and $\mathrm{Rb} / \mathrm{Zr}$ shows that most of the Cam Thuy basalts plot along the mantle array separating the mantle source and continental crust fields (Figs. 13a, b, 14) (after Hoang and Uto, 2003). Note that some of the Song Da low-Ti basalts plot in the crustal field, suggesting, to some extent, crustal contamination. Taken together with the $\mathrm{Sr}$ and $\mathrm{Nd}$ chondritic isotopic compositions (Fig. 10) and OIB-like trace element distribution patterns (Figs. 8 and 9), the Cam Thuy basalts reported here are certainly free from crustal contamination. These geochemical features are also observed for Song Da high-Ti basalts reported elsewhere (e.g. Hoang et al., 2016a), suggesting close similarity in source of origin and melt generation parameters between these two basaltic magmas.

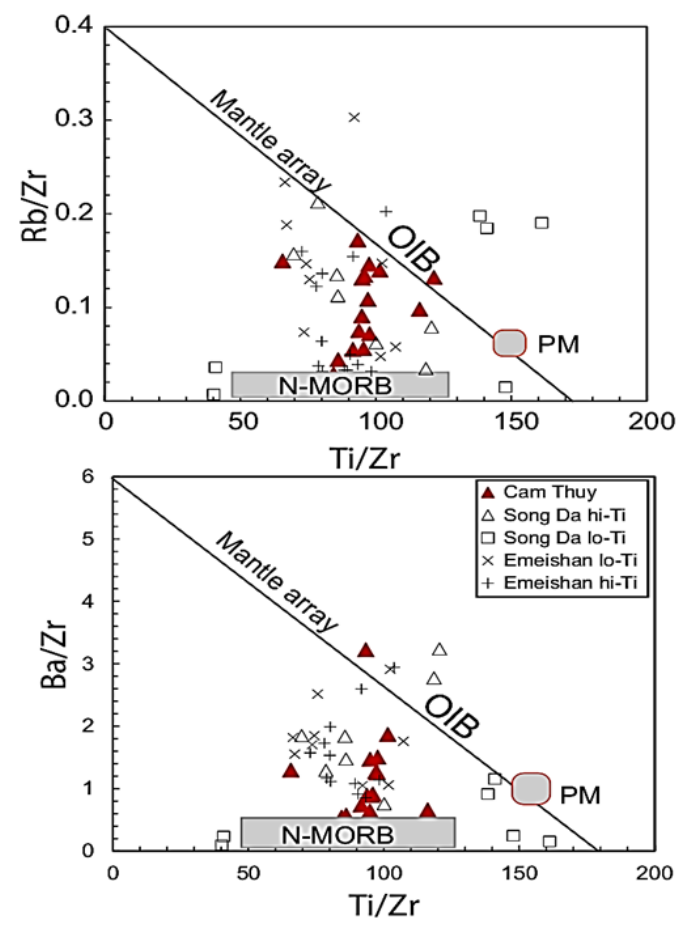

Figure 13. Correlation between (a) $\mathrm{Ti} / \mathrm{Zr}$ and $\mathrm{Ba} / \mathrm{Zr}$ and (b) $\mathrm{Ti} / \mathrm{Zr}$ and $\mathrm{Rb} / \mathrm{Zr}$ for Cam Thuy basalts as compared to Song Da basalts relative to depleted mid-ocean ridge basalt mantle (N-MORB), the mantle array (representing by oceanic island basalt: OIB, after Kogiso et al. (1997) and Frey et al. (2000), and primitive mantle after Hofmann (1988). Modified after Hoang and Uto (2003). See text for explanations 
Vietnam Journal of Earth Sciences, 38(4), 372-392

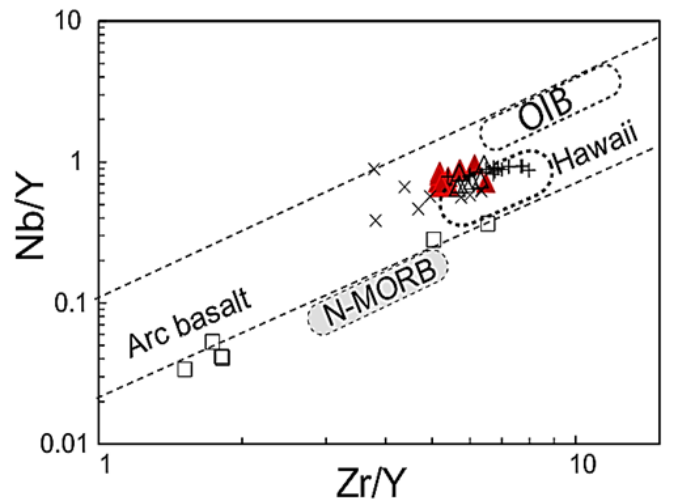

Figure 14. Plots of $\mathrm{Zr} / \mathrm{Y}$ against $\mathrm{Nb} / \mathrm{Y}$ of Cam Thuy basalt along with Song Da and Emeishan magmatic rocks; fields of OIB (North Arch, data from Frey et al., 2000), Hawaiian OIB (after Norman and Garcia, 1999), N-MORB and Arc magmas are shown for reference. Dashed lines signifying ranges of mantle-derived magmas are compiled from world literature

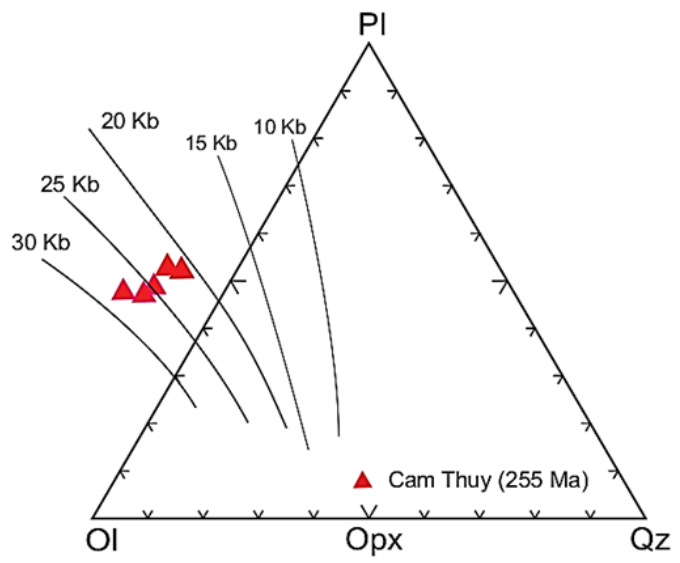

Figure 15. Plots of Olivine $(\mathrm{Ol})$ - Plagioclase $(\mathrm{Pl})-$ Quartz (Qz) for representative computed Cam Thuy primitive melt compositions (after Walker et al., 1979) compared with experimental isobaric liquidi from Hirose and Kushiro (1993) and Kushiro (1996). Accordingly, Cam Thuy basalts segregated from their magma sources between 22.5 and $28 \mathrm{~Kb}$ with potential temperatures of about $1400^{\circ} \mathrm{C}$ to $1450^{\circ} \mathrm{C}$ (after Hoang and Flower, 1998)

\subsection{Mantle sources and melt forming conditions}

Geochemical compositions of lithospheric mantle-derived rocks commonly have high
$\mathrm{MgO}$ and $\mathrm{SiO}_{2}$ contents but especially low $\mathrm{FeO}, \mathrm{CaO}, \mathrm{Na}_{2} \mathrm{O}$ and $\mathrm{K}_{2} \mathrm{O}$ (termed as mafic component), as a result of previous partial melting events (Turner and Hawkesworth, 1995). Generally, the lithospheric mantlederived mafic melts are low in trace element compositions, especially highly incompatible elements such as $\mathrm{Rb}, \mathrm{Ba}, \mathrm{K}$ and light rare earth elements such as $\mathrm{La}, \mathrm{Ce}$ and $\mathrm{Nd}$. However, depending on the timing of melting events (long enough for radioactive decay to form sizable daughter products), cumulating melts from deeper mantle or extracting melts due to local melting could lead to enrichment or depletion of trace element or isotopic composition that may be different (Menzies et al., 1987; Carlson and Irving, 1994; Ionov and Hofmann, 2007). Several studies have suggested that lithospheric mantle is refractory in mafic component and "dry", therefore it is difficult for partial melting to occur. However, Gallagher and Hawkesworth (1994) showed that water liberation from water-rich minerals (such as amphibole) (Ionov and Hofmann, 2007) along with hot mantle upwelling following a lithospheric extension event may facilitate melting processes to occur easily (Turner and Hawkesworth, 1995; Nguyen Hoang and Flower, 1998).

Cam Thuy basalt are relatively low in $\mathrm{MgO}$ and $\mathrm{SiO}_{2}$ contents, high $\mathrm{FeO}, \mathrm{CaO}$ and $\mathrm{Al}_{2} \mathrm{O}_{3}$ although moderate in $\mathrm{Na}_{2} \mathrm{O}$ and $\mathrm{K}_{2} \mathrm{O}$ (Figs. 6, 7; Table 1). As mentioned above the trace element and rare earth element distribution patterns of Cam Thuy basalts are essentially oceanic island basalt (OIB)-like (Figs. 8, 9) in terms of BHVO-2, a Hawaiian OIB standard and JB-1a, a continental intraplate basalt, whose melts are viewed as asthenosphere- derived.

There are several approaches to estimating magma segregation depths. These include: (1) mathematical inversion of melt compositions of specified source and its sub-solidus residua, 
Nguyen Hoang, et al./Vietnam Journal of Earth Sciences 38 (2016)

assuming fractional or batch melting within a polybaric melt column (e.g. McKenzie and O'Nions, 1991; Scarrow and Cox, 1995; Turner and Hawkesworth, 1995), (2) interpolation from $\mathrm{H}_{2} \mathrm{O}$-saturated and unsaturated experimental studies of fertile and refractory peridotite (e.g. Hoang and Flower, 1998). Assuming mantle $\mathrm{H}_{2} \mathrm{O}$ contents beneath NW Vietnam to be minimal we have made best estimates of pressure, temperature and melt fraction by comparing primitive melt compositions with anhydrous or nearanhydrous experimental studies (e.g. Hirose and Kushiro, 1993; Kushiro, 1990, 1996), the approach adopted by Hoang and Flower (1998). The primitive melt compositions used were interpolated from high $\mathrm{MgO}$ basalts, with possible effects of olivine fractionation minimized. Assuming that realistic $\mathrm{Mg} /(\mathrm{Mg}+$ $\mathrm{Fe}^{2+}$ ) ratios of olivine in mantle residua approximate 0.70 , having equilibrated with segregated partial melts. This was achieved by adding small $(0.1 \%)$ increments of olivine to eruptive composition with $\mathrm{MgO}>6 \mathrm{wt} \%$, assuming olivine being the sole liquidus phase observed (Yamashita et al., 1996; after Walker et al., 1979). It was likewise assumed that magmas with mafic phenocrysts of around $\mathrm{Fo}_{89-90}$, match residual mantle olivine, according to an olivine-melt $\mathrm{K}_{\mathrm{d}}(\mathrm{FeO} / \mathrm{MgO})$ value of 0.30 and $\mathrm{Fe}_{2} \mathrm{O}_{3}$ to be 0.15 of $\mathrm{FeO}^{*}$ (Roeder and Emslie, 1970). Plotted in the pseudo-ternary (normative) Ol-Pl-Qz system (Fig. 15; after Walker et al., 1979) estimated primitive Cam Thuy melt compositions are shown for comparison with experimental isobaric, partial melts of spinel/garnet lherzolite (Hirose and Kushiro, 1993; Kushiro, 1996). In the case of Cam Thuy, the calculated melts may reflect a decrease in melt fraction with increasing pressure (22.5 $\rightarrow 28$ Kbar) suggesting a polybaric partial melting column between depths of 65 and 85 $\mathrm{km}$. According to the approach adopted, comparison of primitive magmas to experimental studies of primitive basalts (Kushiro, 1996) and relatively fertile peridotites (HK-66 in Hirose and Kushiro, 1993), potential temperatures of about $1400^{\circ} \mathrm{C}$ $\rightarrow 1450^{\circ} \mathrm{C}$ appear to be reasonable with primitive melts segregating at pressures between 22.5 and $28 \mathrm{Kbar}$ (after Hoang and Flower, 1998), and the most plausible melting mechanism being adiabatic decompression of ductile asthenosphere.

\subsection{Geochemical - petrographic comparison between Cam Thuy, Song Da and Emeishan magmatic rocks}

Song Da magmatic rocks are categorized into high-Ti and low-Ti types. Low-Ti rock type is lower in $\mathrm{Nb}$, Ta, lighter rare earth elements and higher in $\mathrm{MgO}$ and $\mathrm{SiO}_{2}$ relative to the high-Ti magma type (Balykin et al., 2010; Xu et al., 2001, 2004). Trace element and isotopic characteristics of the Cam Thuy basalts are essentially oceanic island basalt (OIB)-like, approximated to Song Da high-Ti basalts (Figs. 8-9). Their initial (255 Ma) Pb, and especially $\mathrm{Sr}$ and $\mathrm{Nd}$ isotopic ratios are chondritic (Figs. 10, 11). The above geochemical and isotopic characteristics along with the computed melting pressures and temperatures Fig. 15), for Cam Thuy basalts and Song Da (in the Son La area) high-Ti basalt indicate that they are most likely derived from an enriched and possibly fertile mantle source (Fig. 14). These features also differentiate the above magmatic rocks from the Song Da low-Ti rock type, which has been considered as heterogeneous lithospheric mantle- derived melts (Figs. 10-14). An analogous type has yet to be discovered among the Cam Thuy volcanic rocks.

The Emeishan high- and low-Ti magmatic types are geochemically heterogeneous, showing highly variable major and trace element contents, covering both Cam Thuy and Song $\mathrm{Da}$ magmatic distribution fields (this study; Xu et al., 2001; Nguyen Hoang et al., 2016a). Their Sr-Nd isotopic compositions 
are also highly variable (Figs. 10, 12-14), trending from depleted to enriched field; although the enrichment, is not comparable to Song Da low-Ti magmatic type, leaving the latter most enriched magma type among the three basaltic regions (Figs. 10-11). A study of picrites in the Emeishan large igneous province suggests a secular change from melting of a peridotite to a garnet pyroxenite mantle source produced, respectively, from the low- and high-Ti magma end-members. Moreover, the similarity in $\mathrm{Sr}$ and $\mathrm{Nd}$ isotopic compositions $\quad\left({ }^{87} \mathrm{Sr}^{86} \mathrm{Sr}_{\mathrm{i}} \sim \quad 0.7045\right.$ and $\varepsilon_{\mathrm{Nd}(t)^{\sim}}$ 1.7) of the two magma types may reflect a source in the sub-continental lithospheric mantle rather than the convective asthenosphere or a deep mantle plume (Kamenetsky et al., 2012).

It has been long believed that Song Da (and Cam Thuy) magmatic association is part of the Emeishan LIP having been extruded southeastward about $700 \mathrm{~km}$ from its SW Chinese site along the Red River Shear zone or an extruding channel between the Red River and Song Ma Fault zone (Fig. 1a) following the India - Asia collision about 30 Ma (Chung et al., 1997; Wang et al., 1997; Lan et al., 2000; Tran et al., 2008; after Tapponnier et al., 1982, 1986; Le Loup et al., 1995; Gilder et al., 1996). While there is not much physical evidence supporting the extrusion mechanism (see Flower et al., 1998; Cung and Geissman, 2013); the fact that the Song Da high- and low-Ti magma types may not be genetically related (Nguyen Hoang et al., 2016a; after Kamenetsky et al., 2012), and that the distribution of Cam Thuy magmatic formation on both sides of the Song Ma fault zone needs further investigation.

\section{Concluding remarks}

Cam Thuy Permian volcanic rocks exposed in the Lam Son area (Tho Xuan, Thanh Hoa province) comprise thick basaltic lava flows and pyroclastic layers, with a total thickness exceeding a few hundred meters.
The rock types are mostly olivine-phyric basalt and a minor amount of alkaline basalt and (quartz-normative) tholeiitic basalt.

The computed melt compositions of the Cam Thuy basalts compared with experimental partial melting of a relatively fertile and enriched spinel lherzolite (HK-66, Hirose and Kushiro, 1993) show their melt segregation pressures from 22.5 to $28 \mathrm{~Kb}$ (ca. 65 to $85 \mathrm{~km}$ ) and corresponding temperatures from $1400^{\circ} \mathrm{C}$ to $1450^{\circ} \mathrm{C}$. Their trace element characteristics are enriched oceanic island basalt (OIB)-like. The major and trace element features along with their chondritic $\mathrm{Sr}-\mathrm{Nd}$ (and $\mathrm{Pb}$ ) isotopic compositions match those of the Song Da (and some of Emeishan) high-Ti magma type, and in conjunction with their geographical proximity, suggesting that they may share a same fertile and thermally anomalous mantle source.

Various low-Ti basaltic and picritic rock types, viewed as melts generated from heterogeneously depleted and refractory sources in the sub-continental lithospheric mantle, appear popular in the Song Da and Emeishan magmatic associations (e.g. Chung and Jahn, 1995; Xu et al., 2001, 2004; Kamenetsky et al., 2012; Tran Trong Hoa et al., 2015) but have yet to be discovered in the Cam Thuy magmatic formation. This difference may reflect discrete source heterogeneity and melting mechanism among the three Permian volcanic fields.

\section{Acknowledgments}

This study is supported by (Vietnam) National Foundation for Science and Technology Development (NAFOSTED) under grant number 105.05-2012-22 (NH). The Institute of Geological Sciences, VAST, is thanked for financing the major and trace element analysis. Tran Thanh Hai, Hanoi University of Mining and Geology, and Phan Van Hung (IGS) are thanked for providing information on the outcrops. Critical 
Nguyen Hoang, et al./Vietnam Journal of Earth Sciences 38 (2016)

comments by two anonymous reviewers help improve the manuscript significantly from an earlier version. Proof reading and suggestions by Rolando Peña (University of the Philippines, Quezon) are gratefully acknowledged.

\section{References}

Anders, E., Grevesse, N., 1989. Abundances of the elements: meteorite and solar. Geochimica et Cosmochimica Acta 53, 197-214.

Balykin, P.A, Polykov G.V., Petrova T.E., Hoang Huu Thanh, Tran Trong Hoa., Ngo Thi Phuong, Tran Quoc Hung, 1996. Petrology and evolution of the formation of Permian-Triassic mafic-ultramafic associations in North Vietnam. J. Geology B/7-8, 59-64, Hanoi.

Balykin, P.A, Polykov, G.V., Izokh, A.E., Tran Trong Hoa, Ngo Thi Phuong, Tran Quang Hieu, Petrova, T.E, 2010. Geochemistry and petrogenesis of Permian ultramafic- mafic complexes of the Jinping-Song Da rift (Southeastern Asia), Russian Geology and Geophysics, 51, 6, 611-624.

Carlson, R.W., Irving, A.J., 1994. Depletion and enrichment history of subcontinental lithospheric mantle: $\mathrm{An} \mathrm{Os}, \mathrm{Sr}, \mathrm{Nd}$, and $\mathrm{Pb}$ isotopic study of ultramafic xenoliths from northern Wyoming Craton. Earth and Planetary Science Letters 126, 457-472.

Chung S.L, Jahn, B.M., 1995. Plume-lithosphere interaction in generation of the Emeishan flood basalts at the Permian-Triassic boundary, Geology, 23, 10, 889-892.

Chung, S.L., Lee, T.Y., Lo, C.H., Wang, P.-L., Chen, C.-Y., Nguyen, T.Y., Tran, T.H., Wu, G.-Y., 1997.Intraplate extension prior to continental extrusion along the Alao Shan-Red River shear zone. Geology, 25, 311-314.

Cung Thuong Chi, Geissman, J., 2013. A review of the paleomagnetic data from Cretaceous to lower Tertiary rocks from Vietnam, Indochina and South China, and their implications for Cenozoic tectonism in Vietnam and adjacent areas. Journal of Geodynamics 69, 54-64.

Dovjikov, A.E. (Editor), 1965. Geology of Northern Viet Nam. General Dept. Geology of Viet Nam. Science and Technique Publishing, Hanoi, 668p (in Russian).
Gallagher, K., Hawkesworth, C.J., 1994. Mantle plumes, continental magmatism and symmetry in the South Atlantic. Earth and Planetary Science Letters, 123, 105-117.

Gilder, S.A, Gill, J., Coe, R.S., Zhao, X.X., Liu, Z.W., Yuan, K.R., Liu, W.L., Kuang, G.D, Wu, H.R., 1996. Isotopic and paleomagnetic constrains $n$ the Mesozoic tectonic evolution of south China. Jourral of Geophysical Research 101, 16,137-16, 154.

Flower, M.F.J., Tamaki, K., Hoang, N., 1998. Mantle extrusion: a model for dispersed volcanism and DUPAL-like asthenosphere in East Asia and the WPAC. In: Flower, M.F.J., Chung, S.L., Lo, C.H. (Eds.), Mantle Dynamics and Plate Interactions in east Asia, Geodynamics Series, vol. 27. American Geophysical Union, pp. 67-88.

Frey, F.A., Clague, D., Mahoney, J.J., Sinton, J.M., 2000. Volcanism at the edge of the Hawaiian plume: petrogenesis of submarine alkalic lavas from the North Arch volcanic field. Journal of Petrology 41, 667-691.

Hanski, E., Walker, R.J., Huhma, H., Polyakov, G.V., Balykin, P.A., Tran Trong Hoa, Ngo, T. Phuong, 2004. Origin of the Permian-Triassic komatiites, northwestern Vietnam: Contributions to Mineralogy and Petrology 147, 453-469.

Hirose, K., Kushiro, I., 1993. Partial melting of dry peridotites at high pressures: determination of composition of melts segregated from peridotite using aggregate of diamond. Earth and Planetary Science Letters 114, 477-489.

Hofmann, A.W, 1997. Mantle geochemistry: the message from oceanic volcanism. Nature 385, 219-229.

Hofmann, A.W., 1988. Chemical differentiation of the Earth: the relationship between mantle, continental crust, and oceanic crust. Earth and Planetary Science Letters 90, 297-314.

Ionov, D., Hofmann, A.W., 2007. Depth of formation of subcontinental off-craton peridotites. Earth and Planetary Science Letters 261, 620-634.

Ishizuka, O., Taylor, R.N., Milton, J.A., Nesbitt, R.W., 2003. Fluid-mantle interaction in an intra-oceanic arc: constraints from high-precision $\mathrm{Pb}$ isotopes. Earth Planet. Sci. Lett. 211, 221-236.

Izokh, A.E., Polyakov, V.G., Tran Trong Hoa, Balykin, P.A., Ngo Thi Phuong, 2005. Permian-Triassic 
Vietnam Journal of Earth Sciences, 38(4), 372-392

ultramafic-mafic magmatism of northern Viet Nam and southern China as expression of plume magmatism. Russian Geology and Geophysics (Geologiya I Geofizika) 46 (9), 922-932 (942-951).

Kamenetsky, V., Chung, S-L., Kamenetsky, M.B., Kuzmin, D.V., 2012. Picrites from the Emeishan large igneous province, SW China: a compositional continuum in primitive magmas and their respective mantle sources. Journal of Petrology 53 (10), 20952113.

Kogiso, T., Tatsumi, Y., Shimoda, G., Barsczus, H., 1997. High $\mu$ (HIMU) ocean island basalts in southern Polynesia: New evidence for whole mantle scale recycling of subducted oceanic crust. J. Geophys. Res. 102, 8085-8103.

Kushiro, I., 1990. Partial melting of mantle wedge and evolution of island arc crust. Journal of Geophysical Research 95, 15929-15939.

Kushiro, I., 1996. Partial melting of a fertile mantle peridotite at high pressure: An experimental study using aggregates of diamond; in: Basu, A., Hart, S.R. (Eds.), Earth Processes: Reading the Isotopic Code. Geophys. Monogr. 95, American Geophysical Union, pp. 109-122.

Lan, C-Y., Chung, S-L., Shen, J.J-S., Lo, C-H., Wang, P-L., Hoa T.T., Thanh, H.H., Martzman, S.A., 2000. Geochemical and $\mathrm{Sr}-\mathrm{Nd}$ isotopic characteristics of granitic rocks from northern Vietnam. Journal of Asian Earth Sciences 18, 267-280.

Le Bas, M.J., Le Maitre, R.W., Streckeisen, A., Zanettin, B., 1986. A chemical classification of volcanic rocks based on the total alkali-silica diagram. Journal of Petrology 27, 745-750.

Le Duy Bach, Dang Tran Quan, 1995. Geological and mineral resources map of Viet Nam 1:200.000. Thanh Hoa Sheet, with Explanatory note. General Dept. Geology of Vietnam, Hanoi.

Leloup H. Ph., R. Lacassin, P. Tapponnier, U. Scharer, Zhong Dalai, Liu Xaohan, Zhangshan, Ji Shaocheng and Phan Trong Trinh, 1995. The Ailao Shan-Red River shear zone (Yuunan, China), Tertiary transform boundary of Indochina. Tectonophysics, 251, 3-84.

Lo, C.H, Chung, S.L., Lee, T.Y., Wu, G., 2002. Age of the Emeishan flood magmatism and relations to Permian-Triassic boundary events. Earth and Planetary Science Letters 198, 449-458.
Menzies, M.A., Rogers, N.W., Tindle, A., Hawkesworth, C.J., 1987. Metasomatic and enrichment processes in lithospheric peridotites, an effect of asthenosphere-lithosphere interaction. In Menzies, M.A., Hawkesworth, C.J. (Eds.) Mantle Metasomatism, 313-359. Academic Press

Nguyen Hoang, Flower, M.F.J., 1998. Petrogenesis of Cenozoic basalts from Vietnam: Implications for the origin of a 'diffuse igneous province'. Journal of Petrology 39, No 3, 369-395.

Nguyen Hoang, Nguyen Dac Lu, Nguyen Van Can, 2004. Paleozoic volcanic rocks in the Song Da region: mantle sources and dynamics. J. Geology 282(7-8, Series A), 10-18 (in Vietnamese with English abstract).

Nguyen Hoang, Shinjo, R., Ogasawara, M., Tran Thi Huong, Phan Van Hung, Flower, M. F.J., 2016 a. Sources of Permian magmas in the Song Da rift zone and their relation to the Emeishan-LIP volcanic rocks (SW China). Lithos (under review).

Nguyen Hoang, Tran Thi Huong, Dao Thai Bac, Nguyen Van $\mathrm{Vu}$, Nguyen Thi Thu, $\mathrm{Cu}$ Sy Thang, Pham Thanh Dang, 2016b. Magma source feature and eruption age of volcanic rocks in the Tram Tau district, Tu Le Basin. Vietnam Journal of Earth Sciences 38 (No 3), 242-255.

Nguyen Hoang, Uto, K., 2003. Geochemistry of Cenozoic basalts in the Fukuoka district (northern Kyushu, Japan): implications for asthenosphere and lithospheric mantle interaction. Chemical Geology 198, 249-268.

Nguyen Hoang, Uto, K., 2006. Upper mantle isotopic components beneath the Ryukyu arc system: Evidence for 'back-arc' entrapment of Pacific MORB mantle. Earth and Planetary Science Letters 249, 229240.

Nguyen Hoang, Uto, K., Matsumoto, A., Itoh, J., 2013. Pleistocene intraplate magmatism in the Goto Islands, SW Japan: implications for mantle source evolution and regional geodynamics. J. Geodynamics 68, 1-17.

Norman, M.D., Garcia, M.O., 1999. Primitive magmas and source characteristics of the Hawaiian plume: petrology and geochemistry of shield picrites. Earth and Planetary Science Letters 168, 27-44.

Pearce, J.A., Norry, M.J., 1979. Petrogenetic implications of $\mathrm{Ti}, \mathrm{Zr}, \mathrm{Y}$, and $\mathrm{Nb}$ variations in 
Nguyen Hoang, et al./Vietnam Journal of Earth Sciences 38 (2016)

volcanic rocks. Contributions to Mineralogy and Petrology 69, 33-37.

Polyakov, G.V., Balykin, P.A., Glotov, A.I., Tran Quang Hieu, Ngo Thi Phuong, Hoang Huu Thanh, Bui An Nien, 1991. Permo-Triassic association of highmagnesian volcanites of the Song Da Zone (NorthWestern Vietnam). Geologiyai Geofizika (Russian Geology and Geophysic) 32(9), 3-15 (1-11).

Polyakov, G.V., Nguyen Trong Yem, Balykin, P.A., Tran Trong Hoa, Hoang Huu Thanh, Tran Quoc Hung, Ngo Thi Phuong, Petrova, T.E., Vu Van Van, 1996. Permian - Triassic mafic and ultramafic formations in northern Viet Nam. Science and Technology Publ., Hanoi, 172 p (in Vietnamese).

Roeder, P.L., Emslie, R.F., 1970. Olivine-liquid equilibria. Contributions to Mineralogy and Petrology 29, 275-289.

Scarrow, J.H., Cox, K.G., 1995. Basalts generated by decompressive adiabatic melting of a mantle plume: a case study from the Isle of Skye, NW Scotland. Journal of Petrology 36, 3-22.

Tang, Q., Li, C., Zhang, M., Lin, Y., 2015.U-Pb age and $\mathrm{Hf}$ isotopes of zircon from basaltic andesite and geochemical fingerprinting of the associated picrites in the Emeishan large igneous province, SW China. Miner Petrol.109, 103-114.

Tapponnier, P., Peltzer, G., Armijo, R., 1986. On the mechanics of the collision between India and Asia. In: M.P. Coward and A.C. Ries (Editors), Collision Tectonics. Geological Society of London, special publication, pp. 115-157.

Tapponnier, P., Peltzer, G., Le Dain, A.Y., Armijo, R., Cobbold, P., 1982. Propagating extrusion tectonics in Asia: New insights from simple experiments with plasticine. Geology, 7, 611-616.

Tong Zuy Thanh, Vu Khuc, 2005. Stratigraphic units of Viet Nam. Vietnam national university publisher, Hanoi, 504pp (in Vietnamese).

Tran Duc Luong, Nguyen Xuan Bao, 1988. Geological map of Viet Nam at 1:500,000. Geological Survey of Viet Nam.

Tran Trong Hoa, 2002. Subdivision and correlation of Permian-Triassic basaltoid association in the Song Da structure (NW Vietnam). Journal of Geology, 19-20.
Tran Trong Hoa (editor), 2011. Intraplate magmatism and metallogeny of North Vietnam. Science and Technology Published, Hanoi.

Tran Trong Hoa, Hoang Huu Thanh, Tran Tuan Anh, Ngo Thi Phuong, Hoang Thi Hang, 1998. High-Ti basaltoid associations in the Song Da rift zone: chemical compositions and formation geodynamics. Journal of Geology, Series A, 244 7-15 (in Vietnamese with English abstract).

Tran Trong Hoa, Izokh, A.E., Polyakov, G.V., Borisenko, A.S., Tran Tuan Anh, Balykin, P.A., Ngo Thi Phuong, Rudnev, S.N., Vu Van Van, Bui An Nien, 2008. Permo-Triassic magmatism and metallogeny of Northern Viet Nam in relation to the Emeishan plume. Russian Geology and Geophysics (Geologiya i Geofizika) 49 (7), 480-491 (637-651).

Tran Trong Hoa, Lan, Ch-Y., Usuki, T., Shellnutt, J.G., Pham, Thi Dung, Tran Tuan Anh, Pham Ngoc Can, Ngo Thi Phuong, Izokh, A.E., Borishenko, A.S., 2015. Petrogenesis of Late Permian silicic rocks of Tu Le basin and Phan Si Pan uplift (NW Vietnam) and their association with the Emeishan large igneous province. Journal of Asian Earth Sciences, 109, 1-19.

Tran Trong Hoa, Nguyen Hoang, 2013. Guide to the field trip. International symposium "Large igneous provinces of Asia: mantle plumes and metallogeny", Hanoi, November 7-11, 2013, 20p.

Tran Trong Hoa, Nguyen Trong Yem, Ngo Thi Phuong, Hoang Huu Thanh, Tran Quoc Hung, Bui An Nien, Hoang Thi Hang, Polyakov, G., Balykin, P.A., Tran, Tuan Anh, 1995. Magnesia-ultrapotassic magmatic rocks and lamproite problems in northwestern Viet Nam. Journal of Geology 5, 412-419 (in Vietnamese with English abstract).

Tran Trong Hoa, Polyakov, G.V., Tran Tuan Anh, Borisenko, A.S., Izokh, A.E., Balykin, P.A., Ngo Thi Phuong, Pham Thi Dung, 2015. Intraplate Magmatism and Metallogeny of North Vietnam. Modern Approaches in Solid Earth Sciences, Springer International Publishing house, Switzerland, 372p.

Tran Trong Hoa, Tran Tuan Anh, Ngo Thi Phuong., Izokh, A.E., Polyakov, V.G., Balykin, P.A., Lan C.Y., Hoang Huu Thanh, Bui An Nien, Pham Thi Dung, 2004. Gabbro-syenite associations of East Bac Bo structures: evidences of intra-plate magmatism? J. Geology, Ser. B 23, pp. 12-25. 


\section{Vietnam Journal of Earth Sciences, 38(4), 372-392}

Tran Van Tri., Tran Trong Hoa, 2011. Late PaleozoicMesozoic Song Da - Tu Le intracontinental rift system. Geology and Natural Resources, Science and Technology Publisher, Hanoi, 425-431.

Turner, S., Hawkesworth, C., 1995. The nature of the sub-continental mantle: constraints from the major element composition of continental flood basalts. Chemical Geology 120, 295-314.

Usuki, T. Lan, Ch-Y., Tran Trong Hoa, Pham, T. Dung, Wang, K-L., Shellnut, G.L., Chung, S-1/., 2015. Zircon $\mathrm{U}-\mathrm{Pb}$ ages and $\mathrm{Hf}$ isotopic compositions of alkaline silicic magmatic rocks in the Phan Si Pan Tu Le region, northern Vietnam: Identification of a displaced western extension of the Emeishan Large Igneous Province. Journal of Asian Earth Sciences 97, 102-124.

Walker, D., Shibata, T., DeLong, D.E., 1979. Abyssal tholeiites from the Oceanographer Fracture Zone III. Phase equilibria and mixing. Contributions to Mineralogy and Petrology 70, 111-125.

Wang, C.Y., Zhou, M.F., Qi, L., 2007. Permian flood basalts and mafic intrusions in the Jinping (SW
China - Song Da (northern Vietnam) district: Mantle sources, crustal contamination and sulfide segregation. Chemical Geology 243, 317-343.

Xu, Y., Bin, H., Chung, S.L., Menzies, M.A., Frey, F.A., 2004. Geologic, geochemical, and geophysical consequences of plume involvement in the Emeishan flood-basalt province. Geology 32, 10, 917-920.

Xu, Y., Chung, S.L, Jahn, B., Wud, G., 2001. Petrologic and geochemical constraints on the petrogenesis of Permian-Triassic Emeishan flood basalts in southwestern China. Lithos 58, 3-4, 145-168.

Yamashita, S., Tatsumi, Y., Nohda, S., 1996. Temporal variation in primary magma compositions in the northeast Japan Arc. The Island Arc 5, 276-288.

Zhou, M.F., Malpas, J., Song, X.Y., Robinson, P.T., Sun, M., Kennedy, K., Lesher, C.M., Keays, R.R., 2002a. A temporal link between the Emeishan large igneous province (SW China) and the endGuadalupian mass extinction. Earth Planet. Sci. Lett.196, 113-122. 